\title{
DIFFERENT SYSTEMS OF KNOWLEDGE FOUND IN THE REPRODUCTIVE HEALTH OF ASHANINKA WOMEN FROM THE PERUVIAN AMAZON ${ }^{1}$
}

\author{
MONIKA KUJAWSKA \\ DEPARTMENT OF ETHNOLOGY AND CULTURAL ANTHROPOLOGY \\ UNIVERSITY OF LODZ
}

\begin{abstract}
Indigenous Ashaninka people from the Peruvian Amazon operate within their communal reserve and autonomous native communities, where biomedical health posts and biomedical practitioners are present. This article analyses how the two systems of knowledge and practice i.e., indigenous medicine and biomedicine, coexist in the Ashaninka territory and how they are articulated in childbirth, birth control and other aspects of reproductive health. Their medicinal cosmology privileges the medicinal plant use and midwifery. At the same time, these practices are compatible with biomedical thinking. The childbirth techniques widely shared by the Ashaninka women and midwifes do not change substantially after biomedical training. Instead, Ashaninka women complementarily add new practices to their own pre-existing array of contraceptive and labour techniques, without shifting their ontological basis. The Ashaninka seem a resilient society, one which is capable of "absorbing" novel biomedical knowledge, practices and technology into their medical landscape, while remaining within their cultural boundaries and thus preserving their specific features.
\end{abstract}

Aszaninka - rdzenna ludność Amazonii peruwiańskiej mieszka i działa w obrębie wspólnotowej rezerwy i w ramach autonomicznych społeczności (hiszp. comunidades), w których znajdują się biomedyczne punkty zdrowia i działają praktycy biomedycyny. Artykuł analizuje, w jaki sposób oba systemy wiedzy i praktyki, tj. rdzenna medycyna i biomedycyna, współistnieją na terytorium Ashaninka i jak są artykułowane w praktykach związanych z porodem, kontrolą urodzeń i innymi aspektami zdrowia reprodukcyjnego. Medyczna kosmologia Aszaninka traktuje ziołolecznictwo oraz położnictwo w uprzywilejowany sposób. Jednocześnie te praktyki etnomedyczne są kompatybilne z biomedycznym sposobem myślenia. Techniki porodu powszechnie stosowane przez kobiety i położne Aszaninka nie zmieniają się znacząco po

I Acknowledgements: The research was conducted within a project financed by the National Science Centre, grant $\mathrm{nr} 2015 / 19 / \mathrm{D} / \mathrm{HS}_{3} / 00632$. The permission for fieldwork and plant collection in Peru was granted by SERFOR, № 252-2017-SERFOR/DGGSPFFS. The author is grateful to the study participants and the CART authorities of the Ashaninka communities from the Tambo River. I am also grateful to Joanna Sosnowska and Miguel Alexiades for reading and commenting on the draft of this paper. 
treningu biomedycznym. Zamiast tego, kobiety Aszaninka rozszerzają swój wcześniej istniejący zestaw technik antykoncepcji i porodu o nowe techniki, nie naruszając zasadniczych podstaw ontologicznych. Społeczność Aszaninka wydaje się być odporna i jednocześnie elastycznie działać (resilience) w obliczu zachodzących zmian w krajobrazie medycznym. Jest w stanie „wchłonąć” nową wiedzę biomedyczną, praktyki i technologię, pozostając w swych granicach kulturowych i zachowując swoje specyficzne cechy.

K e y w o rds: medical pluralism, medical landscape, resilience, medicinal plants, midwifery, indigenous Amazon

\section{INTRODUCTION}

In the ethnographic literature the Ashaninka have been described as a resistant society; one which has managed to adapt to the diverse "historical solicitations" while always preserving the features enabling them to identify themselves as members of Ashaninka society. Historical memories of their struggle and resistance are, beyond human tragedy, symbols of Ashaninka identity and a historical metaphor (Sahlins 1995). During the pre-conquest period, the Ashaninka served as middlemen in the trade of goods between lowland Amazon and the highlands, never losing their independence from the Incas (Heckenberger 2005). They knew also how to be flexible in front of the conquerors, missionaries, and landowners, adapting to the new conditions. They resisted the call of subversive groups such as sendero luminoso and cocaine producers, instead organising themselves into self-defense groups (Span. autodefensa). Nowadays they are in the process of engaging themselves in the modern economy by cultivating several cash crops. This ability to maintain their ethnic identity, together with a high degree of resilience and flexibility in social organisation, can explain, at least in part, their successful resistance to the aggression of the colonial and national powers (Varese 2006, 30-31).

The Ashaninka case offers a complex picture of medical landscape in which we find, I) indigenous Ashaninka medicine, which is not only a referential source of home and expert healing but also a form of articulation of Ashaninka identity and a powerful form of resistance (see derivative function of medicine - De Burgos 20I4); 2) biomedicine with its modernist idea of progress and improvement (Baer 2003). And yet Ashaninka everyday dwelling in the world is intimately related to the knowledge and use of plants. This paper will attempt to demonstrate that the use of medicinal plants by the Ashaninka goes beyond symptomatic response to health conditions, instead it shows strong people, animal and plant relations and interactions. Under ontological scrutiny, the Ashaninka can be labeled as an animist society, the one which has established social relations with non-humans, namely animals, plants, rocks, water bodies and, what we call in Western naturalist ontology - spirits (Descola 1996). However, their social relations with other human groups and non-humans are better explained 
within Amerindian perspectivism framework (Viveiros de Castro 1998). According to this paradigm and to Ashaninka experience, plants and animals are endowed with not only senses and instincts, but also with intensions and agency and all non-humans see themselves as humans.

Medical pluralism (Baer 2003; Crandon-Malamud I99I; Johannessen and Lázár 2006) is a useful framework to understand people's array of possibilities from which one can make his/her medical choices. Medical landscape is another concept, which, according to Kołodziejska (2016, 155)

"better defines the complexity of everyday behaviour (...), because people do not deliberately choose one option from the bunch of possibilities, but they rather act in accordance with the relations they have with, e.g. other people, institutions, medicines, medicinal plants, political power and many others".

The medical pluralism highlights choices people have and uses they can make of them, while medical landscape or health landscape / healthscapes (see Gold and Clapp 20II) pays attention to biophysical and social space in which the interaction of various medical systems take place. In my opinion, both frameworks are suitable to explore the relations between different system of knowledge and accompanying practices in the reproductive health of Ashaninka people. I would like to pay attention to both health-seeking practices of Ashaninka people, choices they make, and also to the coexistence of two parallel systems of indigenous and biomedical knowledge and technology. I hypothesise that these systems are manifested with unequal strength and within unequal power relations. My question here is whether this coexistence of two knowledge traditions in birth control and childbirth may be socially resilient.

Social-ecological resilience has been defined as the capacity of a system to absorb changes and still remain within its boundaries or maintain the same stable state (Holling 200I). Social resilience is often understood intuitively and different definitions exist. The core idea is that societies experience threats, risks and challenges which inevitably lead to responses. These responses may bring adaptation, transformation and change. Resilience is measured in the capacity of a given society to: I) cope with risks and overcome/adjust to adversities, 2) learn from the previous experiences for the sake of future threats, shocks or uncertainties, 3) transform, which encompasses people's ability to obtain assets and help from government organisations and/or civil society, to participate in decision making and to create institutions that increase their agency in the future crises (Keck and Sakdapolrak 2013). It should further be noted that in intercultural contexts resilience does not imply a merely adaptive process or a passive incorporation of foreign cultural elements. A resilient society is one which is capable of "absorbing" disturbances and crisis via a complex re-arrangement of the previous equilibrium. Resilience is related to risks, shocks, and unforeseen unrest, and therefore in medical anthropology has been used mainly in the analysis of health risk 
(Leipert and Reutter 2005; Obrist, Mayumana and Kessy 20I0), but not only (see e.g. Zank, Araujo and Hanazaki 2019). I will attempt to show that it can be useful too in explaining indigenous peoples' capacity to absorb and react towards changes induced by the presence of biomedicine in their communal territories, for example, in dealing with biomedical obstetricians in locally rooted practices such as birth control and childbearing.

To sum up, the aim of this contribution is to describe medical landscape of the Ashaninka in respect to their reproductive health. The description and analysis of the Ashaninka choices may be slightly biased by the fact that I conducted the research mainly from medical-ethnobotanical perspective. By using the concept of resilience, my scrutiny revolves around power relations between Ashaninka indigenous medicine and biomedicine. Finally, I attempt to answer the question whether the coexistence of two systems of knowledge related to family planning and childbirth leads to the biomedical practice becoming Ashaninka (Span. neologism ashaninkar) or does the Ashaninka way of doing things acquire a biomedical way of thinking.

\section{THE ASHANINKA ETHNIC GROUP AND THE STUDY AREA}

The Ashaninka ethnic group belongs to the Arawak linguistic family. The Ashaninka groups as a whole constitute one of the largest remaining indigenous Amazonian groups in Peru. They inhabit the areas along the Apurímac, Ene, Perené, Tambo and upper Ucayali rivers and their tributaries in the region called Selva Central. Groups of Ashaninka are also found throughout Gran Pajonal highland savannas and the eastern bank of the Pachitea river (Varese 2006). As such their settlements and living style show a degree of diversity that ranges from scattered bands to large settled communities along the riverbanks which are well connected to Peruvian society (Killick 2009, 702-703).

The Ashaninka Communal Reserve is located along the Ene, Tambo and Apurímac river valleys and it covers 184,468 ha of tropical highland forest in Peru's central Amazon (Caruso 20II, 6ro). According to Peru's Protected Areas Law no. 26834 of 1997, these are "areas destined for wildlife conservation for the benefit of neighbouring rural populations" (art. 22(g), after Caruso 20II, 608). Communal reserves were set up at the request of indigenous groups wishing to secure territories, both ancestral and for current use (Caruso 20II, 608). These territories are co-managed between the state and the local indigenous groups. Ashaninka people have managed to capitalize on this land category in addition to the comunidad nativa (native community), recognized in the Ley de Comunidades Nativas no. 20635 (1974). The creation of native communities increased the importance of the formal leadership, such as presidente de la comunidad, asamblea general, etc. 
For Ashaninka people, as for other Arawak groups the main unit of society is the nuclear family: one married couple with their unmarried children (Johnson 2003, after Killick 2009). According to my field observations the examples of polygyny and polyandry are extremely rare. Ashaninka people have shown a preference for uxorilocal residence especially in the first years after marriage (Varese 2006). However, over time even those houses connected by close kinship ties move further and further apart (Killick 2009). Since the emergence of native communities and legal rights to their own territory, the Ashaninka enacted a rule, which claims that in the case of a mixed marriage between a non-Ashaninka outsider and an Ashaninka man or woman, the one who takes a non-Ashaninka as a partner must leave the community and live outside the Ashaninka territory. This new law of "traditional" promotes Ashaninka racial, linguistic and cultural uniformity.

\section{METHODS}

The information was collected during three stays among the Ashaninka people, who live along the Tambo river, in 2016, 2017 and 20I8. During the first field campaign in 2016 I worked together with Joanna Sosnowska, PI of the project. The material was collected in the following ways. First, walks to the forest, fallow agricultural areas and agriculture fields (Span. chacras) were taken, where Ashaninka people showed and described the use of medicinal plants. During the course of the fieldwork, 30 walks of this kind were made. Secondly, medicinal plants in 60 Ashaninka home gardens were catalogued. Thirdly, I interviewed is specialists/experts of Ashaninka traditional medicine: midwives, bone setters, shamans and steam-bath makers or other healers (curanderos). Other semi-structured interviews were conducted among lay Ashaninka and experts and concentrated on illnesses and forms of treatment, which could be useful for the analysis of health seeking behaviours. These interviews were complemented with talks with doctors (general practitioners and obstetricians) and nurses in each visited village/community. Altogether 90 adult Ashaninka people participated in the project $(\mathrm{N}$ respondents $=90)$ : 60 women and 30 men, living in Io different communities along the Tambo river. During the fieldwork, I lived with different families in communities sharing their daily routine. The chosen native communities were: Otica, Oviri, Anapate, Poyeni with its three hamlets: Shikapaja, Savareni and Selva Verde, then Vista Alegre, Capitiri, Charauaja, Shevoja, Marankiari and Chembo. They are situated along the Tambo river, a tributary of the Ucayali river, in the tropical Selva Central, Peru.

Herbarium specimens of each mentioned plant was collected - provided it was in a flowering or fruiting phase - then press-dried and deposited in the Herbario de San Marcos del Museo de Historia Natural (USM) in Lima. In the plant identification 
participated Joaquina Albán Castillo, our Peruvian collaborator. The permission for collecting herbarium specimens was granted by SERFOR, № 252-20I7-SERFOR/ DGGSPFFS. This permission also concerned the issue of conducting fieldwork among the Ashaninka people. In order to obtain it, the Ashaninka had to sign an informed consent form. The project itself did not go through any ethics commission either in Poland or in Peru, as the data were based on voluntary interviews with adults.

\section{MEDICINAL LANDSCAPE OF THE ASHANINKA}

I will refer to Ashaninka medicine interchangeably as indigenous or traditional medicine. The latter is a more formal version of the former one, recognized by international bodies, such as World Health Organization. Both indigenous and traditional medicine may be distinguished from biomedicine as autonomous, although likely to have incorporated certain practices from the Western medicine (Gold and Clapp 20II, 94).

For the Ashaninka people home medicine and the expert level of treatment are very much intertwined and rest on a shared ontological basis. The home sector of traditional medicine is a part of an egalitarian stream of knowledge and practice related to health prophylaxis and treatment. Home medicine implies the use of medicinal plants, animal parts, minerals, and magical practices, but not formulas/charms. Practices from expert spheres, like bone setting, massages and midwifery are also part of home medicine. The difference between these two sectors is not a total behavioural change but rather the extent and intensification of the performed healing treatment. However, shamans and steam bath makers have more knowledge and power than lay Ashaninka people to counteract the malevolent spirits (Ash. kamari) and evil caused by other people. A shaman (Ash. sheripiari - a tobacco eater) is a healer par excellence in this society. In reproductive health, the sheripiari plays a limited role. He may be consulted on when a couple cannot conceive a baby and sometimes a shaman is also approached during the pregnancy to recommend an adequate diet. Steam bath makers (Ash. shipokantatsiri; Span. evaporadora) are usually women who share some aspects of apprenticeship with shamans - especially months or even years spent on a special diet and observing sexual abstinence. Their role in reproductive health is minimal - pregnant women may be given steam-baths as a form of prophylaxis or to diagnose a certain health problems. Some women combine bone setting or massage with midwifery. In practically every community there are between one and three midwifes who help pregnant women and assist in the delivery. There exists a gradient between lay midwifes, who help women in their immediate surrounding and the capacitated ones - who are willing to serve the whole community. The Ashaninka midwifes do not keep accounts of the number of pregnant women they helped or the number of labours they assisted, but when asked of how many labours they had assisted the previous year, four to five childbirth were 
mentioned. This relatively low number can be explained by the fact that midwifery is a service that is free of charge, but not a full time job. Five midwifes I spoke to had attended at least one workshop with doctors, called capacitación. Training workshops have been organized by both international NGOs, such Medicos sin Fronteras or Save the Children, but also by the Peruvian Ministry of Health with financial help of Repsol YPF (a Spanish oil company) and by the Centro Amazónico de Antropologia y Aplicación Práctica (CAAAP). Among the capacities gained during these workshops, Ashaninka midwifes enumerated: administering injections, checking of the fetus position, examination of the placenta, and general hygiene protocol. Post-workshop the midwifes introduced subtle modifications to their work, especially related to hygiene. However, these training sessions did not substantially influence their obstetrical knowledge and practice. Indeed, knowledge content of the workshops was secondary to what they learned through experience and from elderly women. Nonetheless, the interviewed midwifes were not disappointed with the courses and workshops. Rather, they felt that their abilities and knowledge had strengthened and had been legitimized through these events. They also felt that they could better understand the doctors' point of view.

As in other developing countries, Peruvian government has put in place biomedical systems not only because of their therapeutic worth but also in the name of social and economic advancement and development (Baer 2003). A similar idea, in the name of progress, have motivated Ashaninka leadership in obtaining funds for constructing adequate infrastructure and keep biomedical staff on their territory. There are three types of health centers (Span. centro de salud) along the Tambo river. Only three centros de salud have a category I-III, in Puerto Ocopa, Poyeni and Betania - the three largest Ashaninka communities. This means that there is one general practitioner there, one obstetrician, one dentist, several (five to eight) nurses and a laboratory technician. Most communities, however, merely have a I-II category health post (posta de salud), which means one obstetrician and two or three technicians and/or nurses. In the smallest communities and hamlets, which have fewer than 200 inhabitants, there is only one technician or health promoter, who has an access to a botiquin - a dispensary with free of charge basic medicines. A doctor pays a visit once a month to these smallest settlements. From this short description, it can be seen that the obstetrician is a privileged biomedical representative in indigenous communities. Their privileged position and high accessibility in indigenous communities stems from a World Health Organization (WHO) policy, which supported the attention given to pregnant women and small children in developing countries. This policy partly stems from the statistics - the Pan American Health Organization results from I990s showed major inequalities in infant mortality and morbidity for indigenous peoples than for national societies. According to these estimates, the Ashaninka had an infant mortality rate of 99 per Iooo infants (Montenegro and Stephens 2006, 1863). Several decades after the implementation of the aforementioned policy, positive results can be seen. Viewed from the statistical point of 
view, the mortality of parturient women and small children has decreased in indigenous groups. On a local level, this policy brought a new agent into indigenous communities - doctor and nurses. On top of this, NGOs supporting indigenous medicine have counterbalanced the efforts of biomedical infrastructure installations in indigenous groups areas by strengthening the role of local health experts and reviving the use and management of medicinal plants. Such actions in Peru have been carried out since the 1990 os by e.g. the Asociación Interétnica de Desarrollo de la Selva Peruana (Aidesep) amongst the Ashaninka, Yinnes and Shipibo (Montenegro and Stephens 2006, I863).

\section{REPRODUCTIVE HEALTH AND THE USE OF PLANTS}

Plants are present in all spheres of the Ashaninkas' life. Their role and their healing properties stem from their position in an overall network of relations between humans and non-humans (see also Lenaerts 2006). The etic division between medicinal and non-medicinal plants should be treated more as an convention made for analytical purposes, rather than a reflection of these people reality. Medicinal plants are often linked to diet and food proscriptions and are used to counteract the malicious behaviour of many different kinds of insects. The latter, especially ants, are seen as troublemakers by the Ashaninka. Many etiological concepts of illnesses are built around malevolent insects. The widely held assumption is that when a husband or a wife has extramarital sexual relations, she/he becomes infected with "bugs" (bichos). Table I contains details about plant use in cases of partner infidelity, as well as information about plant pusangas - love potions related to love magic (see also Figure I).

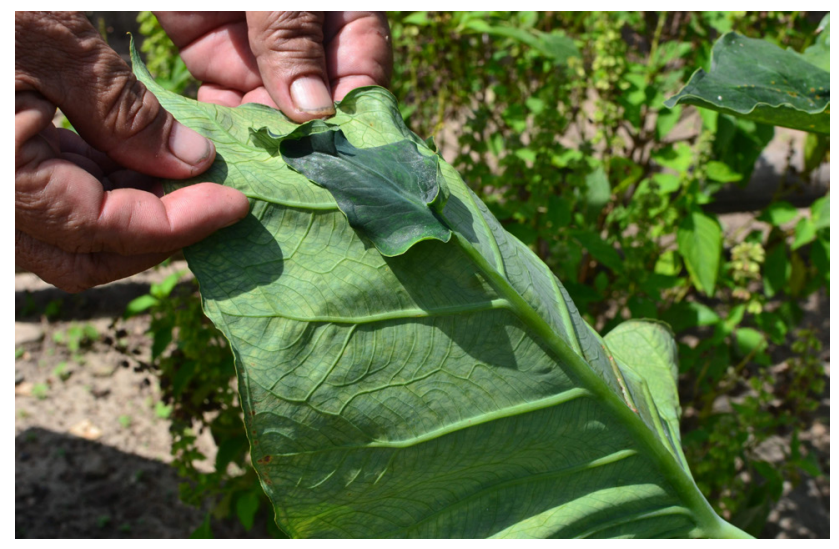

Figure I. Leaves of Xanthosoma cf. sagittifolium which resemble vagina. The starchy tuber of this plant is used as a pusanga (photo: M. Kujawska). 


\section{A proper diet}

Ashaninka people understand that the body is built through an adequate diet and physical activity. The idea of adequate food permeates the whole life of these people, a feature characteristic to other Amerindian groups, who have processual approach to becoming and being a person (e.g. Århem 1996; Rival 2002). Here I will just point to the diet and food proscriptions during gestation and childcare. Other food proscriptions relevant to Ashaninka reproductive health can be found in the Table I.

Pregnant women put greater attention to food taboos than they do to a balanced diet, so promoted by nurses and teachers in recent years (starting from early age at school). Here it is important to introduce a Spanish term cutipar, which means to copy, gain or assimilate the characteristic of an other. In most cases these are animals and plants which can cutipar, i.e. a person gains the characteristic of a given species of animals or plants. Especially unborn children and infants are vulnerable to being cutipado (passive form of the verb cutipar) by animals and plants. The general idea is that a child will take the characteristic of fruit or animals, which his/her mother ate while being pregnant. Therefore, pregnant women, especially from the seventh month of gestation onward, are very careful with what they eat. Once I stayed with a couple whose youngest son had not starting walking by the age of four. According to the parents, that might have been due to the fact that his mother had eaten water turtle when she was pregnant with him. The parents seemed helpless and the child had not had any medical tests or shamanic examinations done. In this situation, manioc, plantains and some species of fish, which constitute food par excellence, is the safest food for pregnant women. However, some women get tempted by game meat, as well as prohibited kinds of fish or fruit, which they should avoid. For these occasions some women cultivate ibenki (Cyperus sp.) and ibinishi (mostly Justicia spp. ) in their home gardens, and given to counteract the effects of the proscribed food. Both ibenki and ibinishi are generic names, in most cases they have their proper names (see Table I). Forest plants are also consumed when a food proscription has been broken. Perinatal dietary and activity restrictions are mostly limited to a pregnant woman. Her husband may be involved, however, and they both perform couvade (see also Rival 1998).

Even more food and activity restrictions are put upon parents of a new-born child most game animals and birds should be avoided. Even a simple activity, such as hunting in the forest may affect the baby, as the spirit of the child accompanies his father. For example there is a small black partridge, called shianti that when the parents eat it, it affects (cutipa) the baby. It makes the child cry, disturbs him/her, and the baby follows its voice. If parents break the diet, and eat this partridge there is a special forest plant, shiantishi (Peperomia sp.) to remedy the situation.

\section{Birth control with plant agents}

The Ashaninka get married when they have the capacity and the confidence to care for themselves and their families. Indeed, the desire to display this ability seem 
to be part of the reason for marrying (Killick 2009; 705). As in other Amazonian societies the main goal of a marriage is to have and raise children (Rival 2002; Cayón 2013). Through child rearing, real and lasting bonds between spouses are built. The average number of children in the families I worked with was $4 \cdot 4$, with a minimum of I (adopted) and a maximum of I2. Couples who have lots of children are the subject of gossip and disdain by their neighbours, who claim that children cannot have a suitably good upbringing in such families.

Ashaninka women have a number of ways to control the number and spacing of children by contraception and abortion and their practice includes the parallel use of natural (plant) and synthetic (biomedical) agents. For example, plants are used in order to delay ovulation and make a woman sterile for a period of time. In these cases, plants are always used at the end of the menstruation cycle. Several species were determined, which were claimed to be effective by the Ashaninka women (Table I). Different authors, describing plant contraceptives used by the Amazonian groups, have taken different positions in deciding whether plants can be effective or not. According to Hern (1994), plants used by indigenous women are not effective - and the fact that these people have relatively few children is due to numerous periods of sexual restraint during their lives. In contrast, both Maxwell (1972) and Belaunde (1997) describe the "sacred plants" used for contraception by indigenous women from the Amazon. Maxwell supports their effectiveness, which is sustained by the women's testimonies, while Belaunde claims that no tests had been performed that could prove their effectiveness. Contrary to the description of Maxwell and Belaunde, the knowledge about contraceptive plants amongst the Ashaninka is not treated as secret or privileged. Apart from the previously mentioned Cyperus sp., whose compounds and properties are described below, the other identified plant used for this event is Pseudelephantopus spiralis. The only known activity of this species is antiplasmodial and antileishmanial (Girardi et al. 2015).

Voluntary miscarriage is also practiced and women talk about it discreetly yet openly. Women claim that plant abortifacients are effective when used in the very early stages of pregnancy because they are not very strong. When a woman finds out that she is pregnant later, she is normally afraid of inducing abortion by the means of plants, which may bring complications to her or to the fetus, and as a result, the child may be born with a defect. On the other hand, those women who cannot get pregnant visit shamans, and steam bath makers and take advice from elderly women. During our walks in the forest I recorded a few plants which were used to improve women's fertility. They are known under an umbrella name bairontsiki. Tectaria incisa and Desmodium adscendens are consumed in a way that a woman may choose the sex of the baby: When long leaves are ingested, it is for a boy (chirampari) and whereas round leaves will result in a girl being born (tsinane) (Figure 2). 


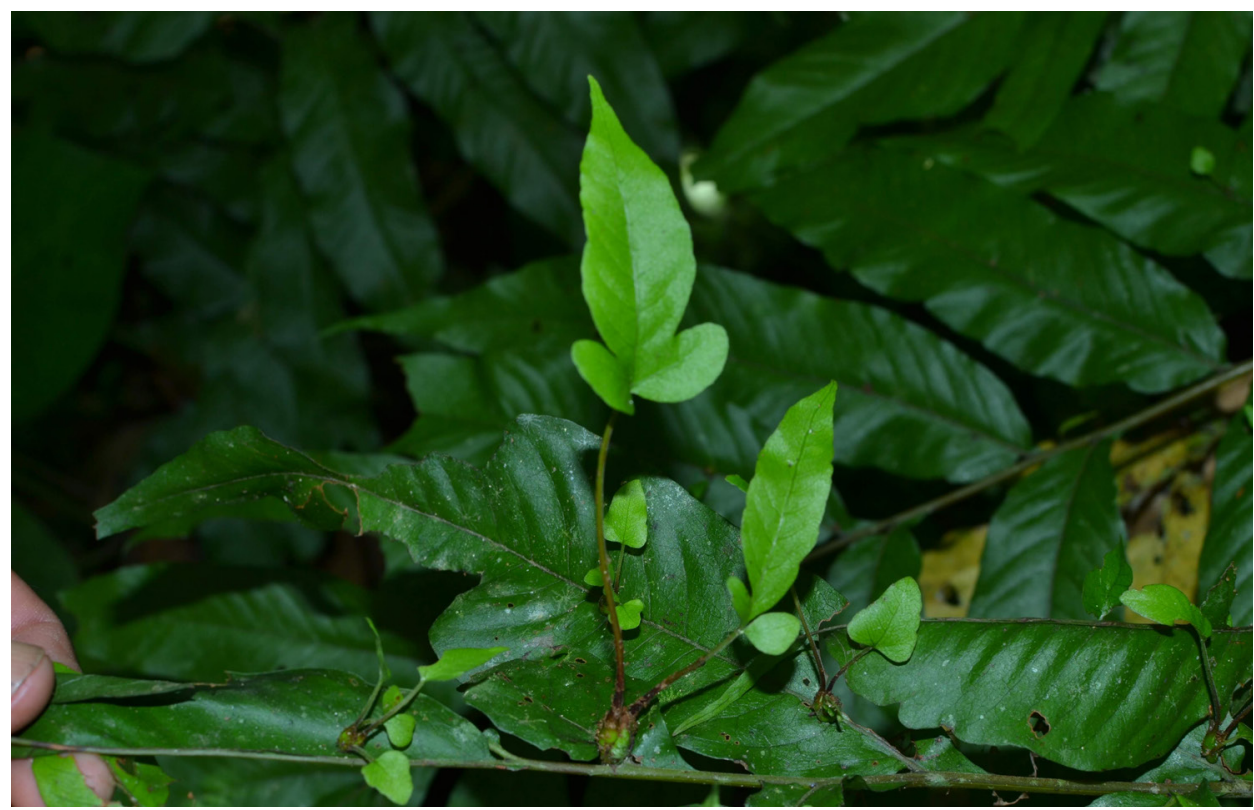

Figure 2. Leaves of ohoairontsiki (Tectaria incise) used to improve women's fertility. They are consumed in a way that a woman may choose the sex of the baby: when long leaves are ingested, it is for a boy (chirampari) and whereas round leaves, it will result in a girl being born (tsinane) (photo: M. Kujawska).

\section{Childbirth}

Ashaninka women find out that they are pregnant when their menstruation does not appear. Other known symptoms are that the nipples become stiff and small, and some marks and spots appear on the face. If the woman wants to keep the baby, she carries on her daily routine.

Women use plants to facilitate the labour or to make it less painful. These plants are both wild species growing in the forest and cultivated in home gardens. Here, I will concentrate on the cultivated plants. The most frequently used ones are sedges (Cyperus spp.), known as ibenki, which have extremely diverse medicinal applications. These ibenki are used to accelerate labour contractions. According to phytochemical findings, this is not the plant itself that produces this effect, but a parasitical fungus found on it (Balansia cyperi) (Plowman et al. 1990). These fungi produce ergot alkaloids, which are most probably responsible for the medicinal properties ascribed to sedges by the Ashaninka. The highest concentration of the fungus is found on rhizomes, and this part is always used by the Ashaninka. It is either chewed, with the juice swallowed, or a decoction is prepared and drunk. Very similar use of ivenkiki has been reported 
among the Matsigenka, belonging to the same Arawak family (Shepard 1998). Another widely used species for soothing pains and accelerating labour is a perennial herb, with the generic name ibinish (Justicia pectoralis). It contains betaine, which is a non-specific anti-convulsive agent (Freed, Gillin and Wyatt 1979). The species contains secondary metabolites, such as coumarin and umbelliferone (Macrae and Towers 1984). At doses approaching toxic level, coumarin has a sedative and hypnotic effect (Macrae and Towers 1984: 107). These properties could explain the persistent use of Justicia spp. during labour.

\section{FERTILITY CONTROL AND CHILDBIRTH FROM THE PERSPECTIVE OF PARALLEL SYSTEMS OF KNOWLEDGE}

As was explained above, Ashaninka women use both plant and synthetic contraceptives. Young women seem to be more inclined towards the use of pills and vaccines than middle aged women. Obstetricians give contraceptive pills every month to young women, due to lower amount of hormones. Women in their late twenties and older may receive a vaccine every three months. Use of intrauterine devices (IUD) is never practiced and doctors explained that women carry heavy loads of manioc and logs, so it could move inside and as a result prove ineffective. While plant contraceptives are used openly, synthetic ones are used covertly, without the consent of a husband, who could be suspicious that his wife is cheating on him.

Ashaninka women reluctantly report vaginal infection to biomedical staff due to the widely shared concept, according to which there exist a direct relation between such infections and extramarital sexual relations. Therefore, women exclusively use medicinal plants for such events. Moreover, women avoid hand examinations by obstetrician as they are seen to be both painful and shameful.

According to obstetricians, women are more and more often visiting health posts or centers to take a pregnancy test. If they are pregnant, they are registered and they attend periodic check-ups. Pregnant women obtain mineral supplements, folic acid and vitamins free of charge from the health posts.

From the seventh month of pregnancy onwards a woman will start checking the position of the baby with the assistance of a midwife or an experienced woman in her family. Everybody is afraid of breech delivery - only the most skillful and experienced midwives are capable of assisting them. Nearly all births occur at home, with only a fraction being performed in a health center or a post where an obstetrician has a dedicated space for births. Caesarean delivery is never performed in any of the health centers of the Tambo river, due to a lack of proper sterile conditions. These centers are poorly equipped - ultrasound scans are never performed, neither are rudimentary blood tests. Obstetricians use a stethoscope and hands to examine the position of the 
fetus. Women do not want to be attended by obstetricians during labour because they reportedly "put their hands inside too much". However, some women do not mind if an obstetrician is present during the domestic delivery, albeit not as the main assistant, but rather as a watchful observer. The main assisting person is more likely to be the mother or a mother-in-law, husband or, in some cases, a midwife. Midwifes are called when they are part of a family or when a woman is left on her own and cannot be assisted by her family, or when she and her partner are inexperienced. Birthing is done in a vertical position and women support themselves with a pole or a rope. Normally the husband stays behind to physically support the woman. The Ashaninka, midwifes, or obstetricians do not make vaginal cuts, but when the vagina is broken, obstetricians or nurses will sew the stitches or, in their absence, a midwife will do so.

From the doctors and nurses' point of view, it is their duty to assist domestic childbirth; if they turn down a request and something goes wrong during the labour, they may be in trouble. Doctors and nurses are generally aware of the plants taken by the woman before the labour begins. Obstetricians claim that these plants are taken wrongly and make parturient women push when the aperture is just six or seven, and so they are already exhausted when the proper labour starts (when the aperture is ten). Therefore, on a declarative level, their aim is to correct the inadequate use of plants by the Ashaninka, not to discourage them from using plants.

From midwifes' point of view, the workshops they have participated in, along with their loose collaboration with biomedical staff in the community, have contributed to an increase in hygiene during labour. Midwifes can get alcohol and gloves from the health post. Once they are in a hut to attend the labour, they insist on using boiled water. Cotton strings used to wrap the naval are also boiled in water. My own findings stay in line with another piece of research performed in low technology birthway scenario in the neotropics (tropics of the New World), and indicate that the influence of biomedicine on midwifes does not substantially alter their obstetric knowledge and practice, which is deeply embedded within their hands-on experience (Sesia 1996).

RELATIONS OF POWER AND RESILIENCE OF ASHANINKA MEDICINE

\section{Streams of legitimized knowledge}

There is a clear distinction between Ashaninka and biomedical practice concerning birth control and childbirth. These two systems dispose of their own means and experts. Nonetheless, the Ashaninka women have a choice and they can pick practices from both systems which are convenient for them: some practices are resisted and others are welcomed. The Ashaninka system of knowledge has a greater legitimacy than biomedicine. It is empowered by the fact that the Ashaninka are on their own land and within their social milieu, where they speak their own language, and share their 
cosmovision and lifestyle. The Ashaninka not only outnumber biomedicinal staff in their communities, but they also make use of two sectors of knowledge transmission and exchange - an egalitarian stream of knowledge based on the recognition and use of medicinal plants, animal and mineral derives, as well as an expert level of knowledge confined to some individuals but based on a widely shared ontological basis. The required plant and animal resources are within easy access, in home gardens, fallow fields and in the forest. On the other hand, biomedical staff rely on only the expert level of knowledge, which may be transmitted in a limited space, i.e. in health posts and health centers. During home childbirth obstetricians and nurses may give limited advice and be of help, however the whole procedure of labour is managed by the Ashaninka.

\section{Between community members and outsiders}

The Ashaninka exhibit a double standard of behaviour and approach towards their own people and outsiders, respectively. They highly prize individual autonomy and harmonious social interactions, which often require curbing personal ambitions and resentments, as was observed in other Arawak societies (Izquierdo 2005). On the other hand, outsiders, represented in Ashaninka communities by teachers and biomedical staff, are approached with a demanding attitude. Although the social interactions with outsiders living in the community tend to be peaceful, nonetheless, the Ashaninka's effort focused towards obtaining measurable benefits from them. Outsiders, after all, are "goods bringers". This way of socialising with outsiders could be perfectly explained from an Amerindian perspectivism conceptual framework (Descola 20I3). However, here I will limit my argument only to the possibilities of knowledge exchange and social interactions, as well as influencing other systems, without diving into the deeper motivations of the Ashaninka.

Ashaninka agency and sovereignty is strengthened during frequent community meetings and social gatherings, during which people discuss what is good and beneficial to them. Doctors and nurses are usually excluded from these social gatherings and are only occasionally invited to community meetings - always as an "external body". There exists a whole protocol which determines the rules of engagement between biomedical staff and the community. It is usually the presidente de la salud (health president) - a person chosen from the community, who acts as an intermediary between the community and the biomedical staff, to plan and organize health campaigns, vaccinations etc.

It is Ashaninka women and their families who decide whether the childbirth will be held at home or not, what kind of contraceptives to use, etc. In these circumstances, it is the obstetricians and nurses who must adapt to the situation to a greater extent than the Ashaninka do. Does the coexistence of two systems of knowledge related to family planning and childbirth lead to the biomedical practice becoming Ashanink 
(Span. neologism ashaninkar) or does the Ashaninka way of doing things acquire a biomedical way of thinking? In my opinion neither occurs. Thanks to the presence of biomedicine in the Ashaninka communities the extent of choices has become greater. Biomedical knowledge, resources, and technology are added to the array of ethnomedical practices, but they do not change the underlying Ashaninka ontological basis. Some practices are omitted - especially hand examinations performed by obstetricians. On the other hand, biomedical staff cannot openly question people for their use of medicinal plants, and neither are midwifes criticized for doing so. Rather, their practices are corrected by placing greater concern on hygiene and people are encouraged to consult on their health problems at an early stage with doctors and nurses, not just with shamans and other experts. These findings are in line with the conclusions of Lenaerts concerning Asheninka ethnomedicine, that

"[t] he borrowing process partially means going toward the Peruvian world (...). The result is a clear proximity to urban people, though keeping very clear indigenous features" (Lenaerts 2006).

\section{Herbalism and midwifery - resilient practices}

Herbalism and midwifery are two elements of the ethnomedical systems in Latin America which are recognized as compatible with biomedical thinking (Heckler 2007; Sesia 1996). According to Heckler (2007), the declining importance of shamanism amongst the Piaroa indigenous group from Venezuela has occurred in parallel to the promotion of herbalism by Christian missionaries and has subsequently been strengthened by biomedicine. Although, I have not conducted a longitudinal study on the use of medicinal plants amongst the Ashaninka, the whole discourse built around medicinal plants is striking. The Ashaninka want to promote their phytotherapy and plan to have a book published, one that would safeguard their intellectual property rights about medicinal plants, and which would bring healing oriented tourism. They thus want to capitalize on their "deep knowledge of plants", as recognized by mainstream western society. Yet at the same they do not express a similar willingness towards promoting their shamanism. There are only a few shamans still active along the whole Tambo river. On the other hand, knowledge of medicinal plants is prominent and complex. Plants are inexpensive, locally available, and embedded in Ashaninka cosmovision and are thus recognized as their own agents of treatment. My findings differ with those of Heckler in the sense that medicinal plants are not used to treat merely symptoms by the Ashaninka but very often, as was shown in this article, are intertwined with deeper ontological relations between humans and non-humans.

Therefore the persistent use of medicinal plants and midwifery are resilient practices - ones which are privileged by Ashaninka medical cosmology, and which at the same time are validated by biomedical practice, especially in the low technology contexts of indigenous communities. 


\section{CONCLUSIONS}

The Ashaninka people operate within their own communal reserve and within their own autonomous communities, which create the conditions for the biomedical practice, which is characterized by low technological input in which doctors, obstetricians and nurses have limited options to exercise their practice. Ashaninka people do not see this as a major obstacle in interacting with biomedical staff. They simply want to maintain relations of power in which it is for them to decide how the methods of birth and birth control will be performed. They see biomedical obstetric practices as complementary to their own. The imposition of relations of power by the Ashaninka epitomises their resistance towards melting with general mainstream society, and their awareness of their distinct cultural features.

Midwifery and labour techniques are widely shared between lay Ashaninka women and expert midwifes. The use of plants is prominent and complex and permeates all spheres related to reproductive health. Although the Ashaninka may see midwifery and herbalism as a distinctive feature of their society, and one which is privileged by their medical cosmovision and supported by the long tradition of trial and error practice, in fact these aspects of their ethnomedicine are also compatible with, and hence validated by biomedicine, which sees them as complementary with their own practice. Perhaps, this is for this reason that the frictions and tensions between biomedical staff and the Ashaninka are almost invisible and oriented towards consensual rearrangements.

The Ashaninka seem a resilient society, one which is capable of "absorbing" novel biomedical knowledge, practices and technology within a complementary approach, while remaining within their boundaries and preserving their specific features. Ashaninka birthways and birth control cope with the presence of biomedicine and with the changes it introduces: the Ashaninka are learning and selectively transforming. 


\begin{tabular}{|c|c|c|c|c|c|}
\hline 芴 & 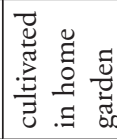 & 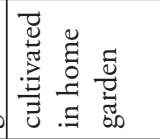 & 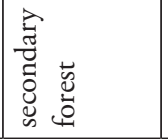 & 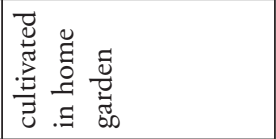 & 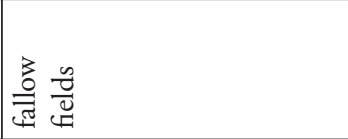 \\
\hline 苛 & 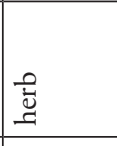 & 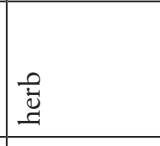 & 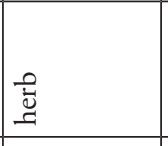 & 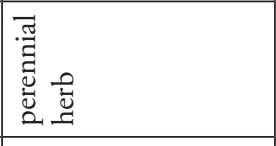 & 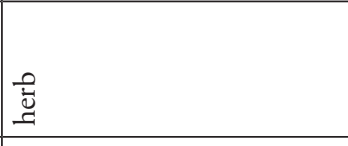 \\
\hline 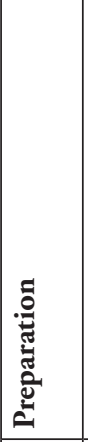 & 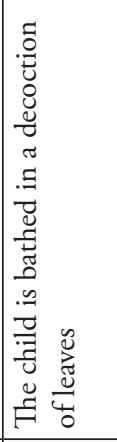 & 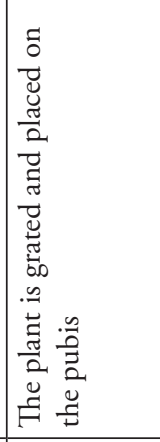 & 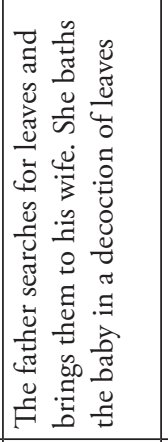 & 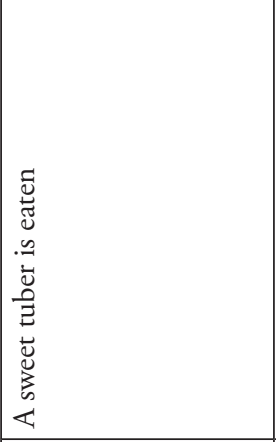 & 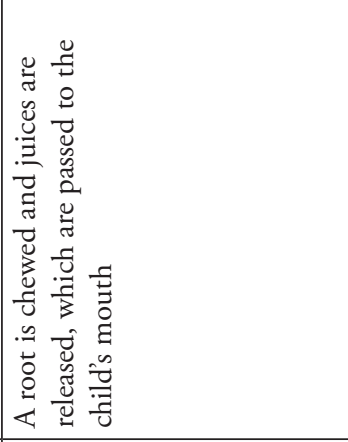 \\
\hline$\stackrel{\mathscr{D}}{D}$ & 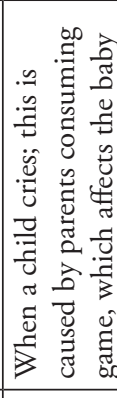 & 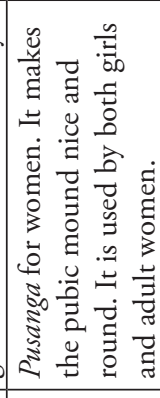 & 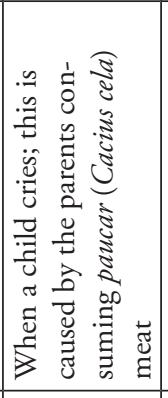 & 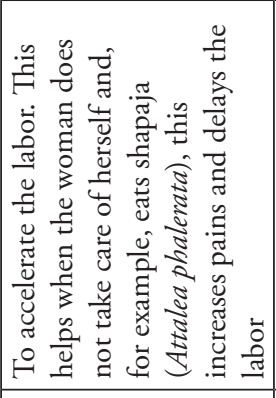 & 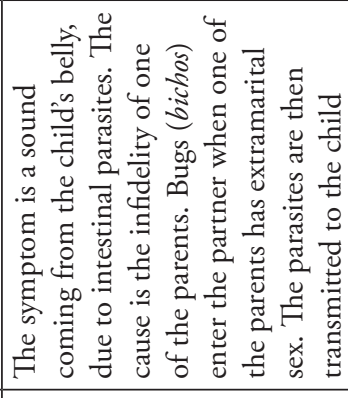 \\
\hline 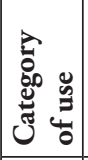 & 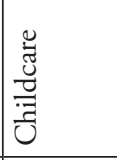 & 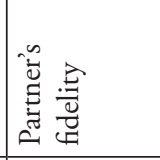 & 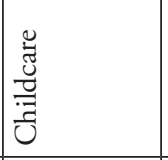 & 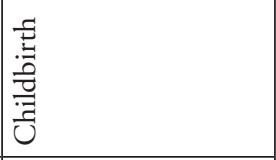 & 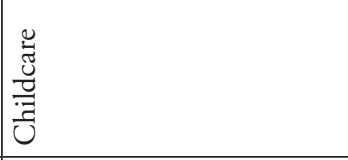 \\
\hline 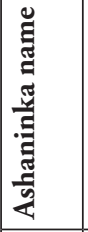 & 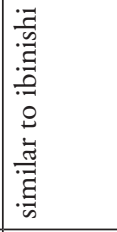 & 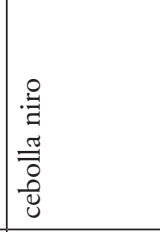 & 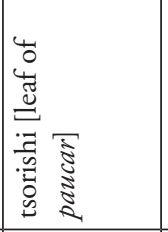 & 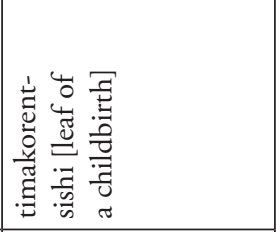 & 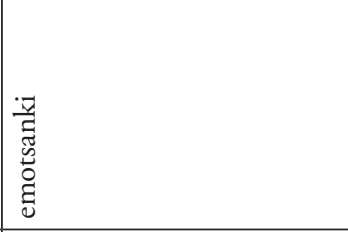 \\
\hline 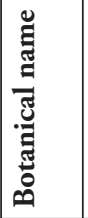 & 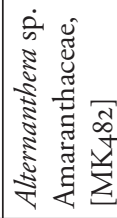 & 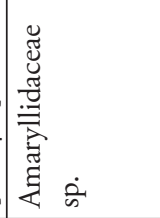 & 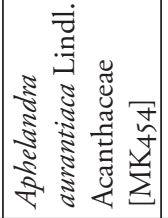 & 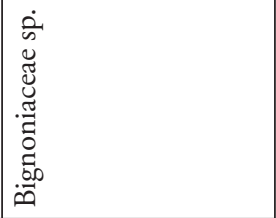 & 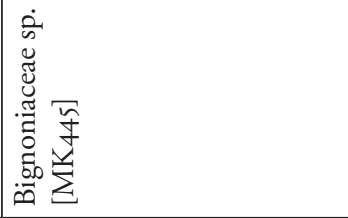 \\
\hline
\end{tabular}




\begin{tabular}{|c|c|c|c|c|c|c|}
\hline 苞 & 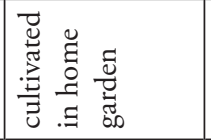 & 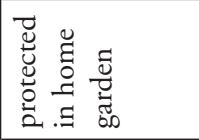 & 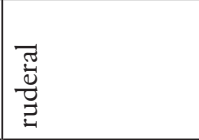 & $\begin{array}{l}\overline{\widetilde{G}} \\
\overline{\vec{U}} \\
\bar{Z}\end{array}$ & 总 & 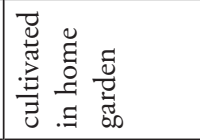 \\
\hline$\stackrel{0}{0}$ & 昙 & 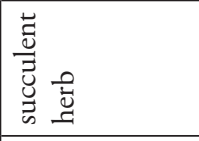 & 司 & 咅 & 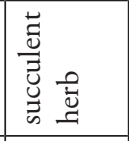 & 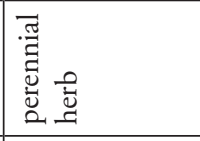 \\
\hline 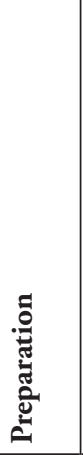 & 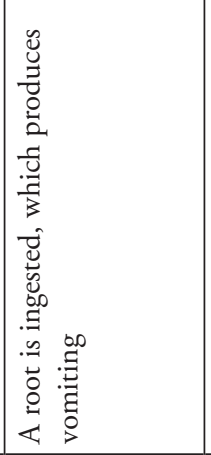 & 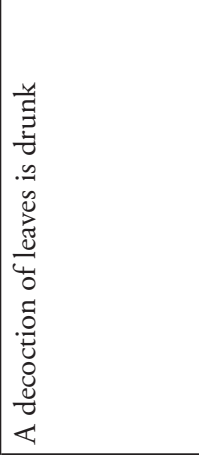 & 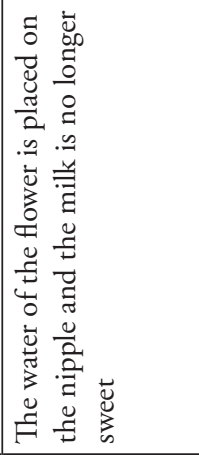 & 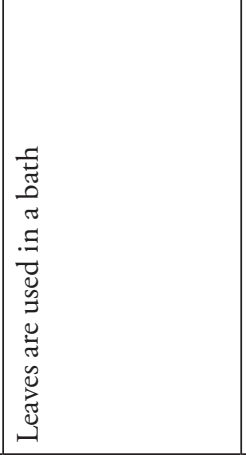 & 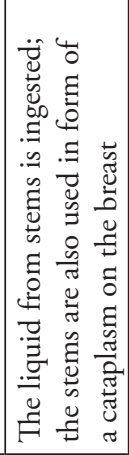 & 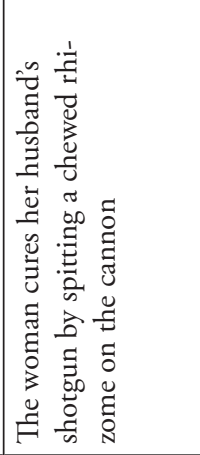 \\
\hline$\stackrel{\mathscr{D}}{.}$ & 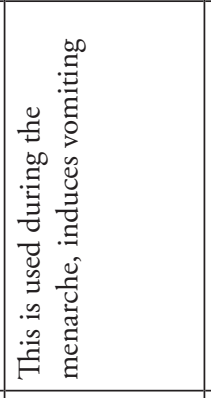 & 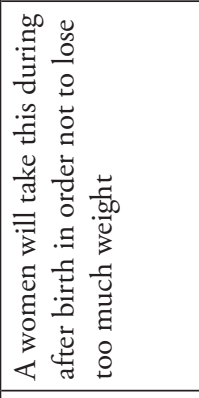 & 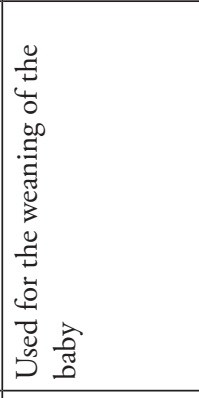 & 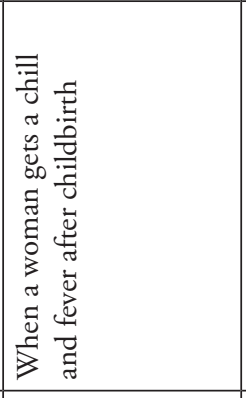 & 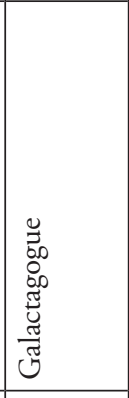 & 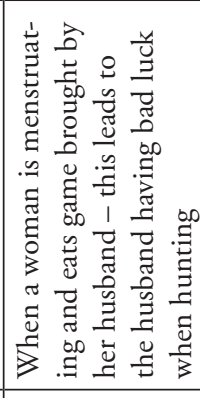 \\
\hline 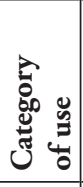 & 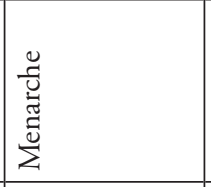 & 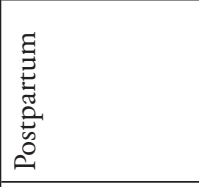 & 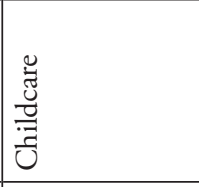 & 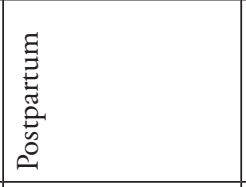 & 节 & 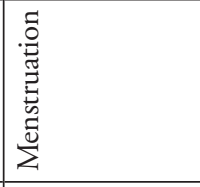 \\
\hline 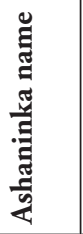 & 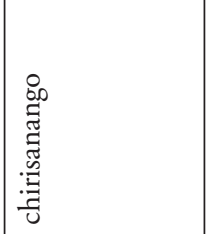 & 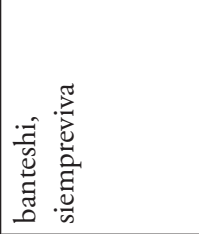 & 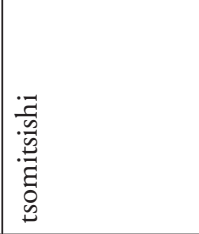 & & 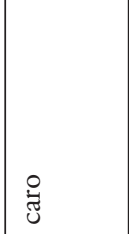 & 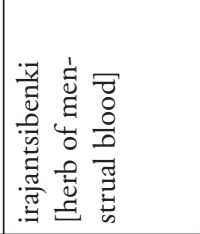 \\
\hline 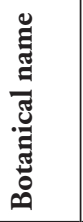 & 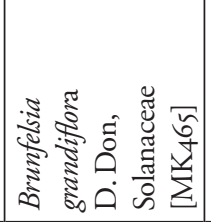 & 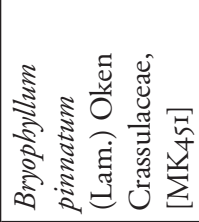 & 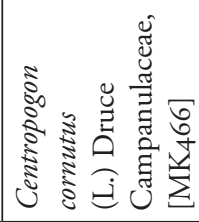 & 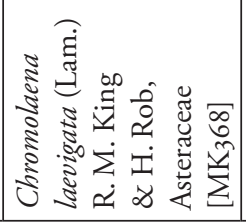 & 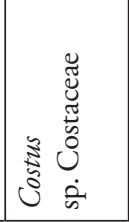 & 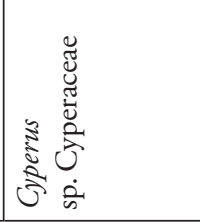 \\
\hline
\end{tabular}




\begin{tabular}{|c|c|c|c|c|c|c|}
\hline 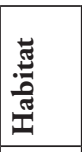 & 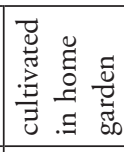 & 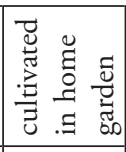 & 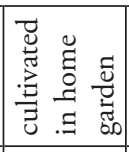 & 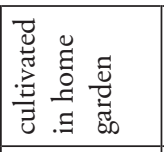 & 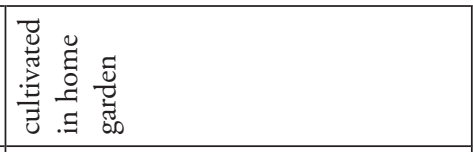 & 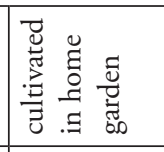 \\
\hline 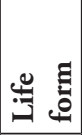 & 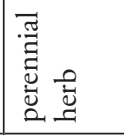 & 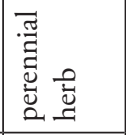 & 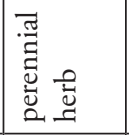 & 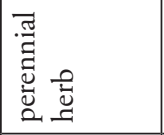 & 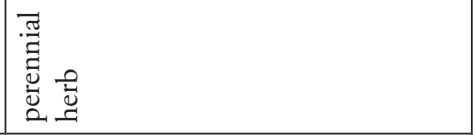 & 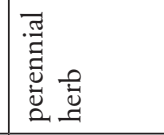 \\
\hline 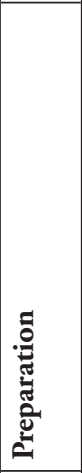 & 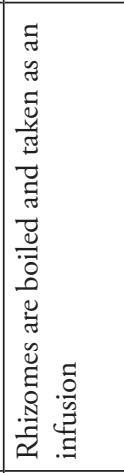 & 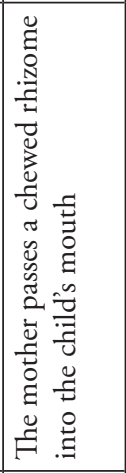 & 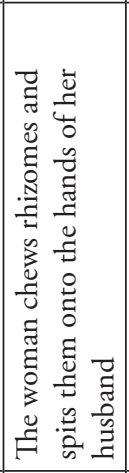 & 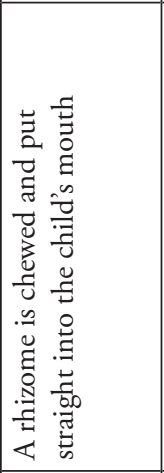 & 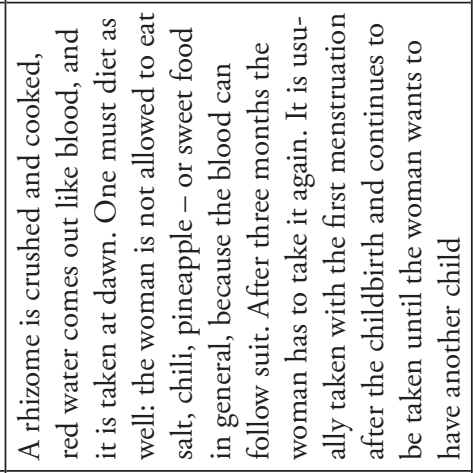 & 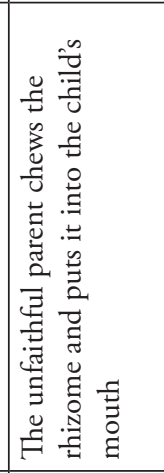 \\
\hline$\stackrel{\mathscr{D}}{\ddot{D}}$ & 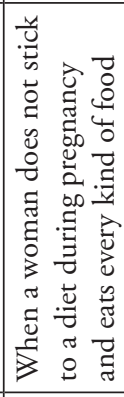 & 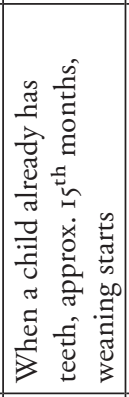 & 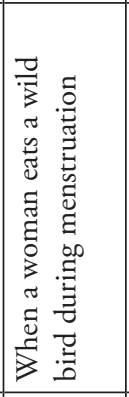 & 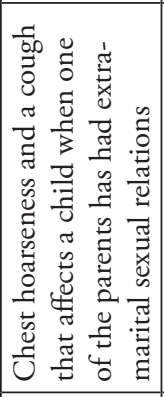 & 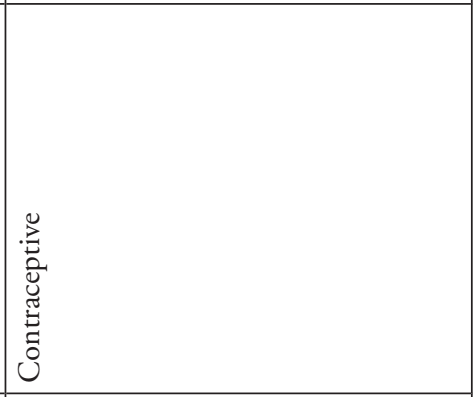 & 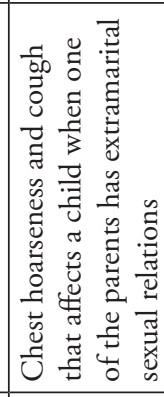 \\
\hline 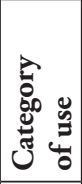 & 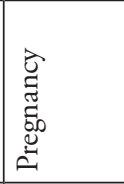 & 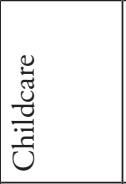 & 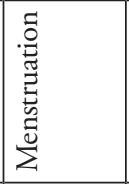 & 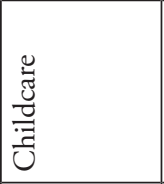 & 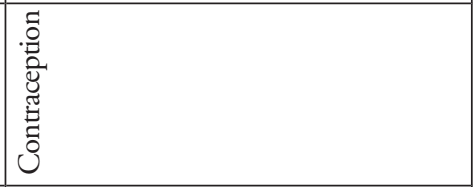 & 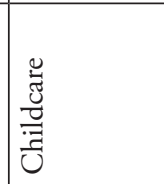 \\
\hline 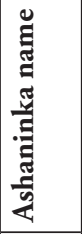 & 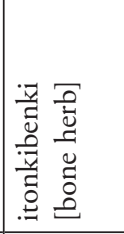 & 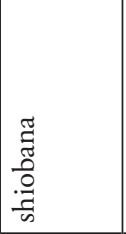 & $\begin{array}{l}\frac{\vec{y}}{\bar{v}} \\
\stackrel{0}{:}\end{array}$ & 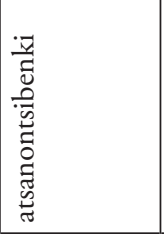 & 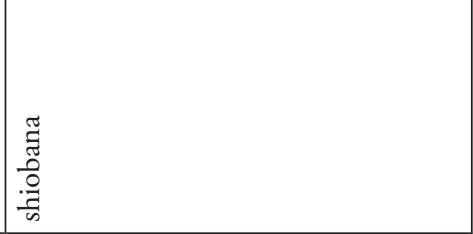 & 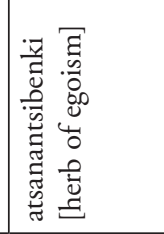 \\
\hline 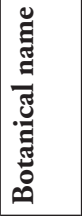 & 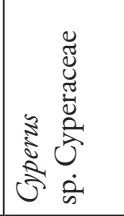 & 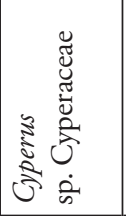 & 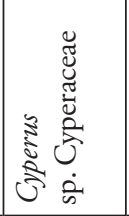 & 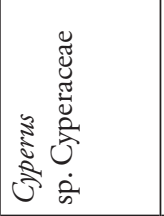 & 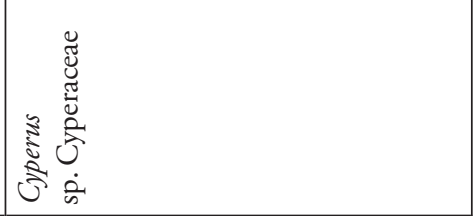 & 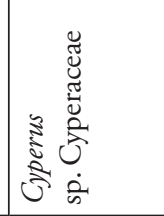 \\
\hline
\end{tabular}




\begin{tabular}{|c|c|c|c|c|c|c|}
\hline 节 & 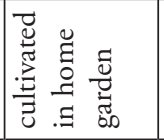 & 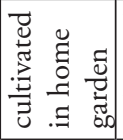 & 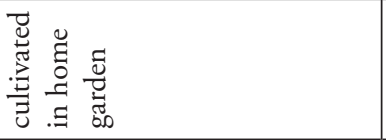 & 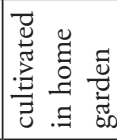 & 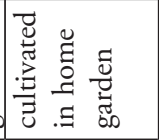 & 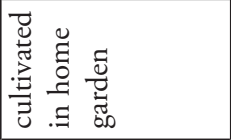 \\
\hline 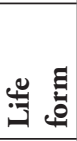 & 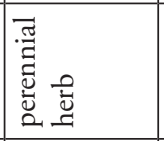 & 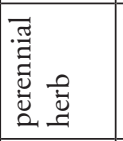 & 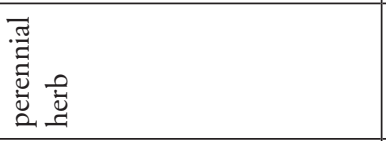 & 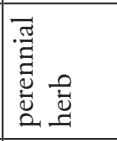 & 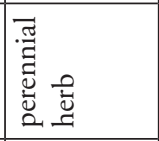 & 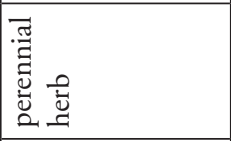 \\
\hline 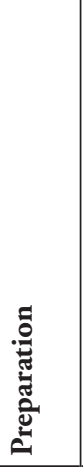 & 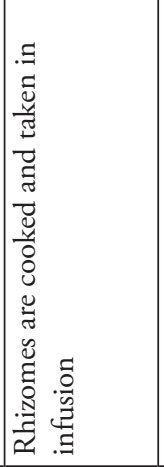 & 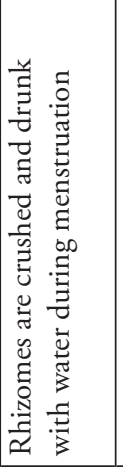 & 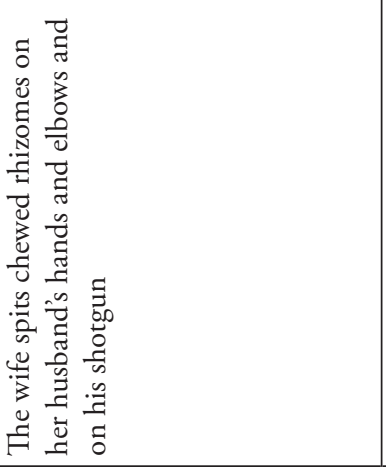 & 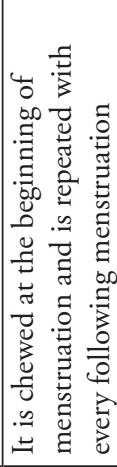 & 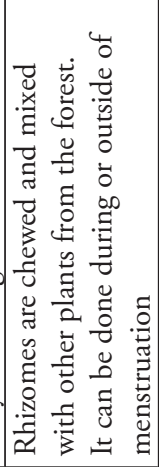 & 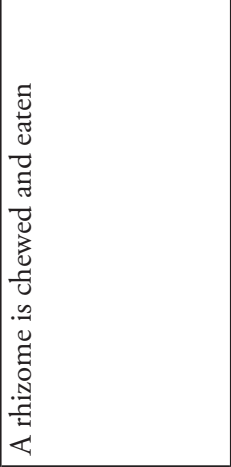 \\
\hline$\stackrel{\mathscr{n}}{D}$ & 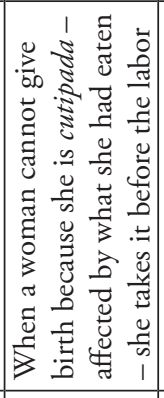 & 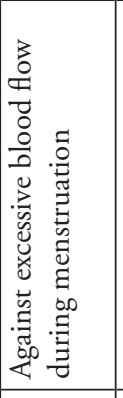 & 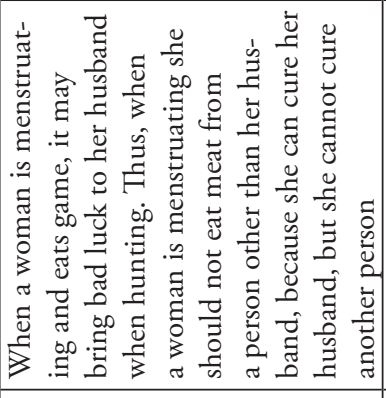 & 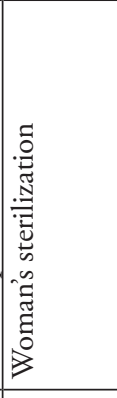 & 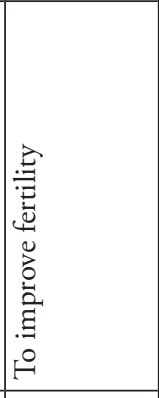 & 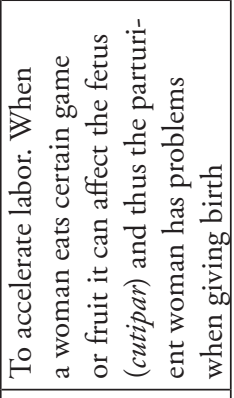 \\
\hline 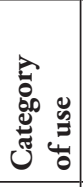 & 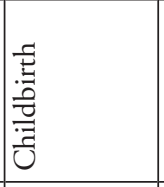 & 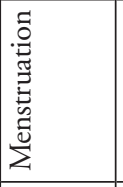 & 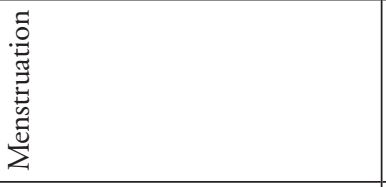 & 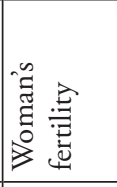 & 告 & 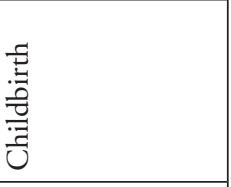 \\
\hline 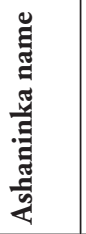 & 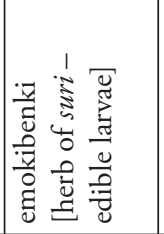 & 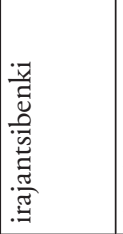 & $\begin{array}{l}\frac{\vec{y}}{\bar{d}} \\
\stackrel{0}{=}\end{array}$ & 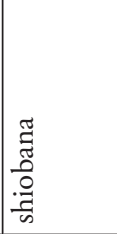 & 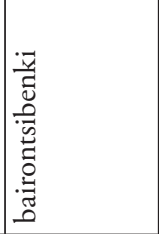 & 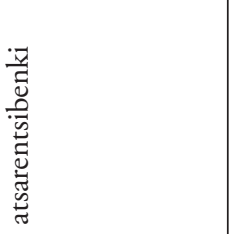 \\
\hline 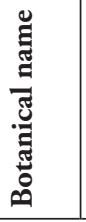 & 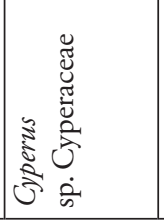 & 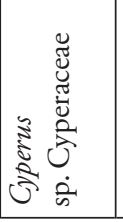 & 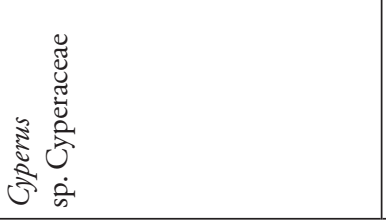 & 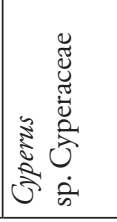 & 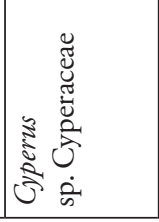 & 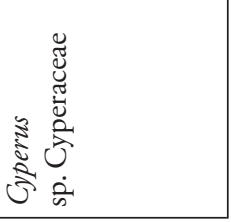 \\
\hline
\end{tabular}




\begin{tabular}{|c|c|c|c|c|c|c|c|}
\hline$\underline{\underline{u}}$ & 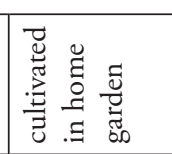 & 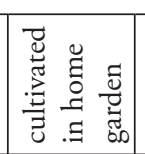 & 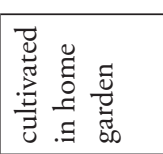 & 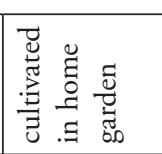 & 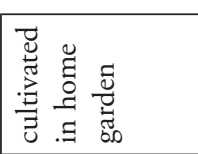 & 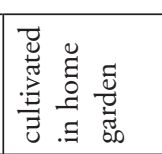 & 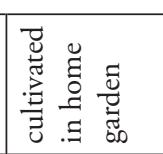 \\
\hline$\dddot{g}$ & 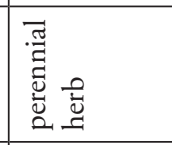 & 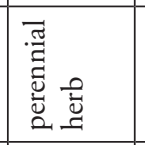 & 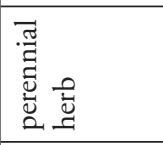 & 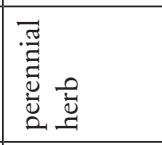 & 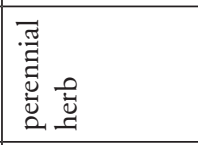 & 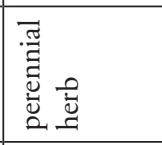 & 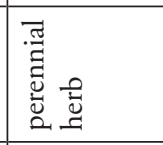 \\
\hline 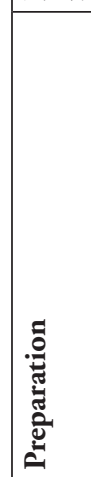 & 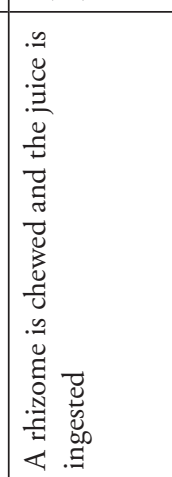 & 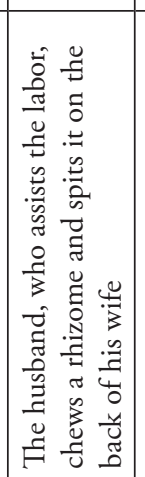 & 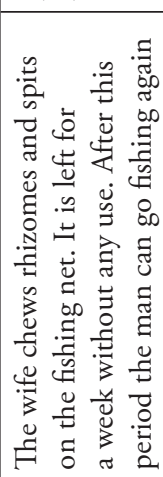 & 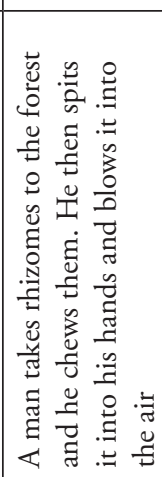 & 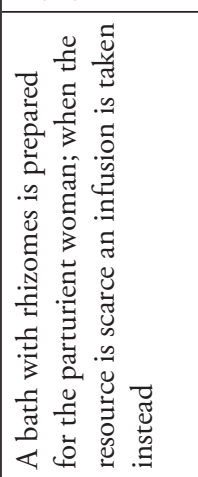 & 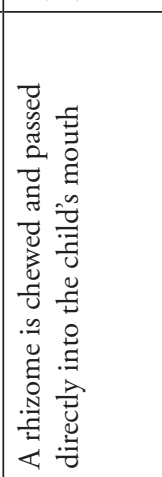 & 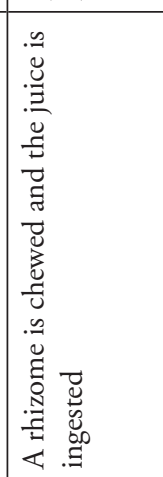 \\
\hline D. & 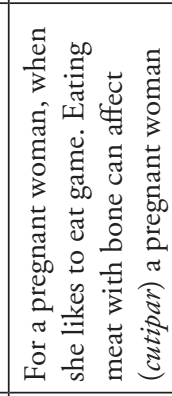 & 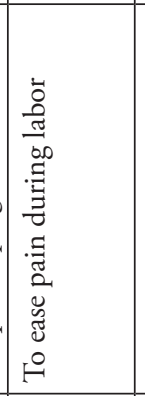 & 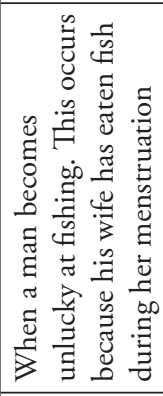 & 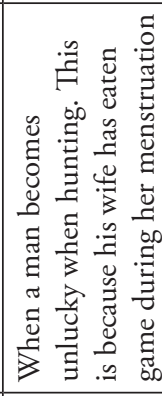 & 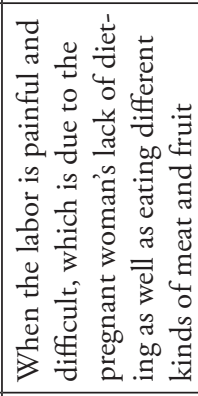 & 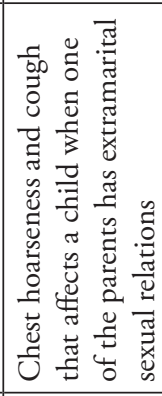 & 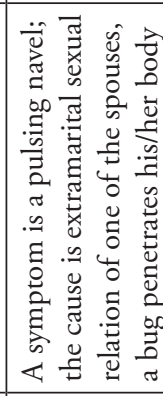 \\
\hline 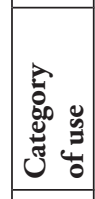 & 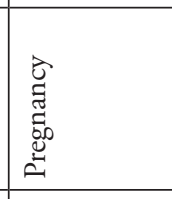 & 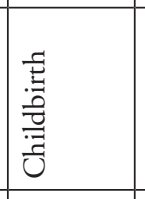 & 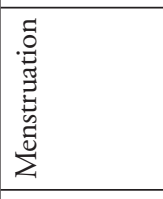 & 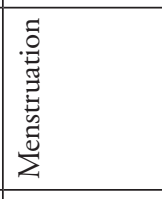 & 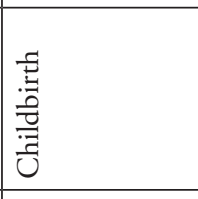 & 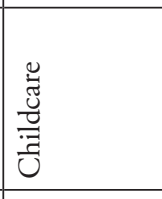 & 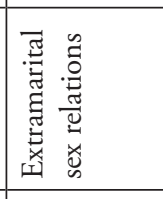 \\
\hline 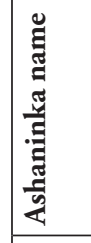 & 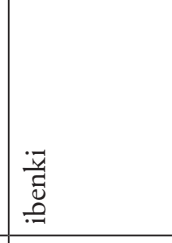 & 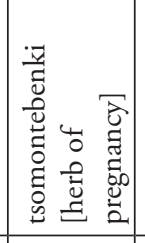 & 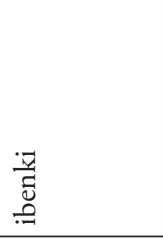 & 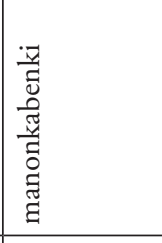 & 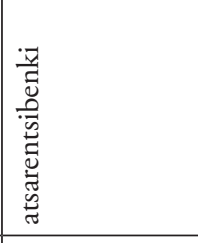 & 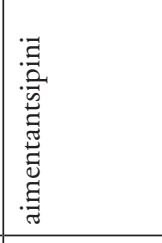 & 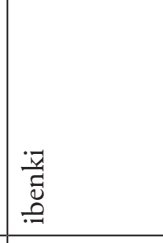 \\
\hline 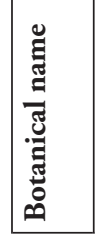 & 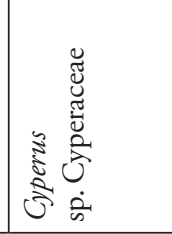 & 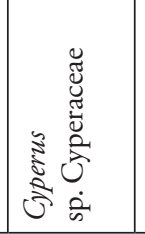 & 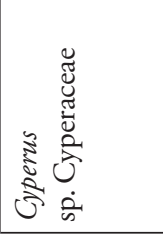 & 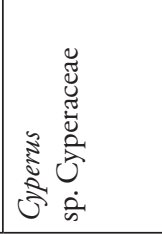 & 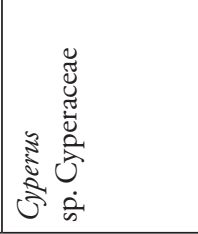 & 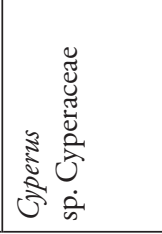 & 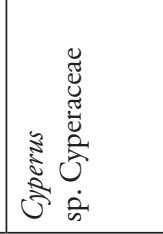 \\
\hline
\end{tabular}




\begin{tabular}{|c|c|c|c|c|c|c|}
\hline 䒕 & 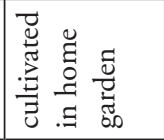 & 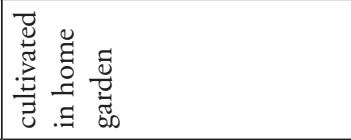 & 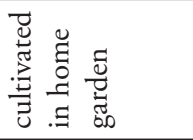 & 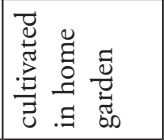 & 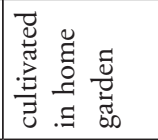 & 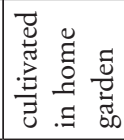 \\
\hline 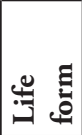 & 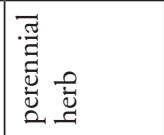 & 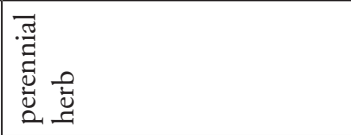 & 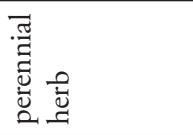 & 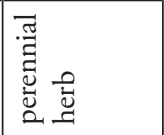 & 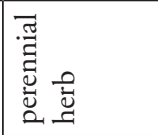 & 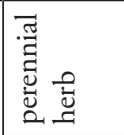 \\
\hline 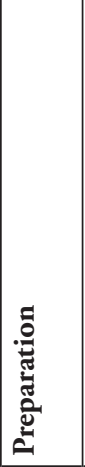 & 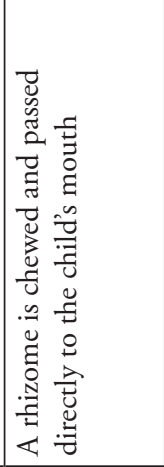 & 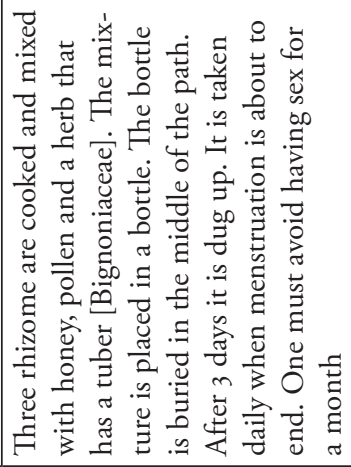 & 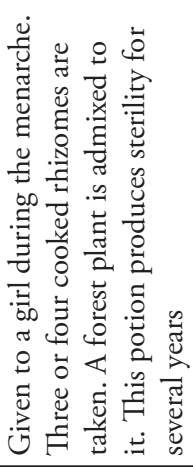 & 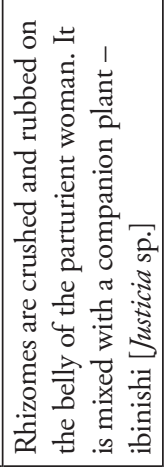 & 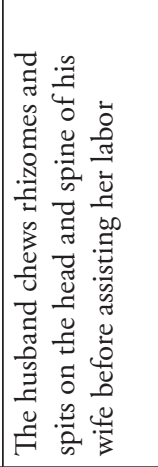 & 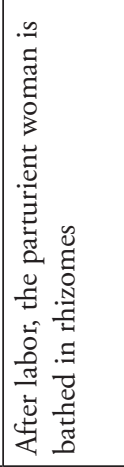 \\
\hline$\stackrel{\mathscr{D}}{\infty}$ & 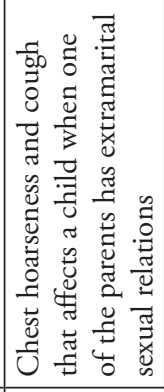 & 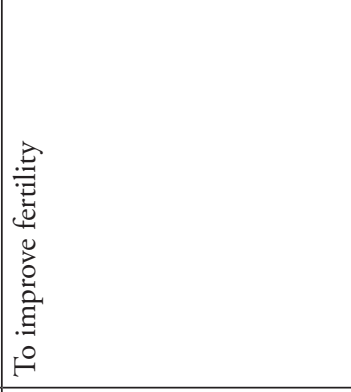 & 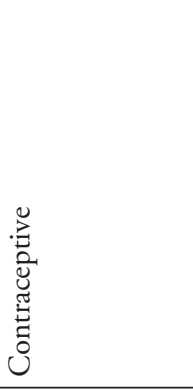 & 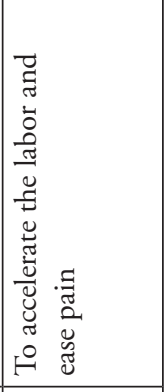 & 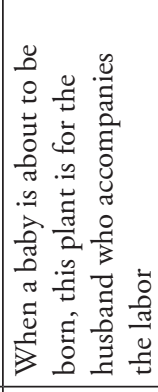 & 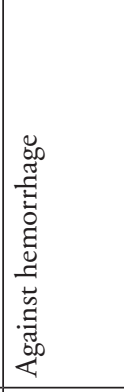 \\
\hline 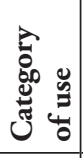 & 䒕 & 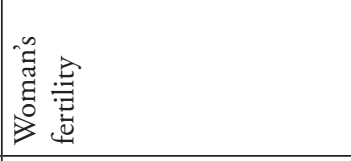 & 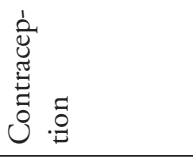 & 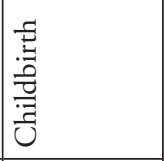 & 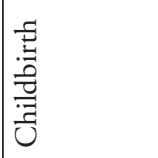 & 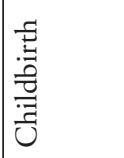 \\
\hline 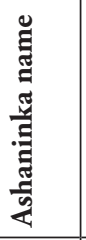 & 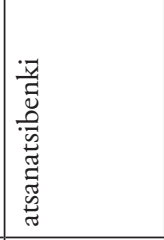 & 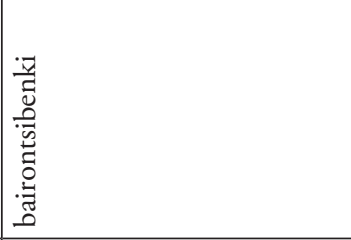 & 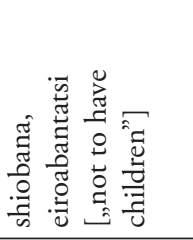 & 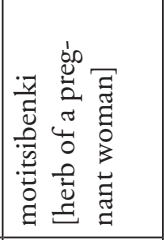 & 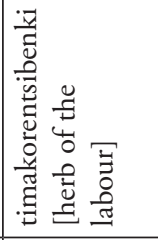 & 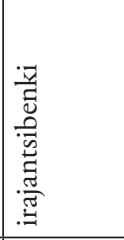 \\
\hline 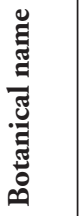 & 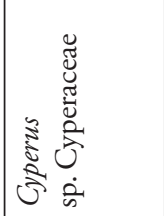 & 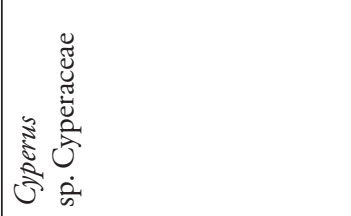 & 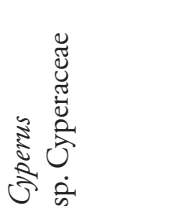 & 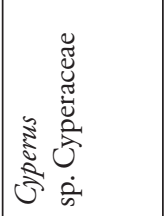 & 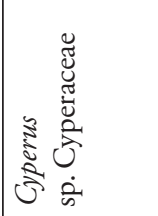 & 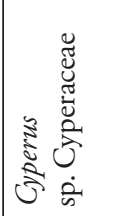 \\
\hline
\end{tabular}




\begin{tabular}{|c|c|c|c|c|c|}
\hline 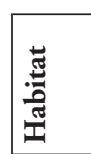 & 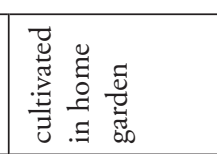 & 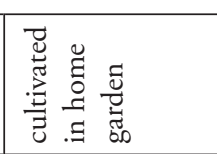 & 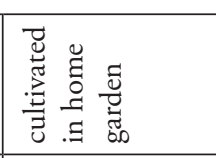 & 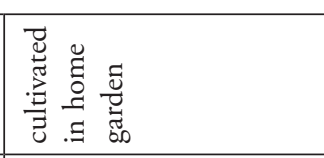 & 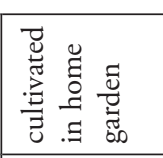 \\
\hline$\dddot{0}$ & 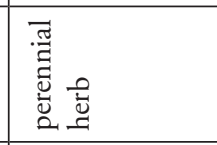 & 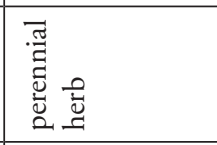 & 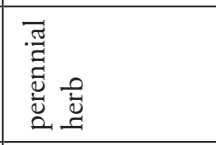 & 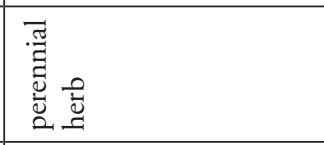 & 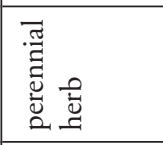 \\
\hline 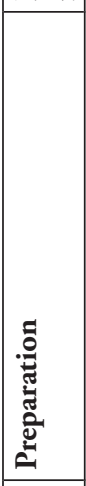 & 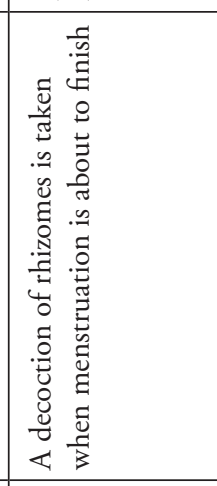 & 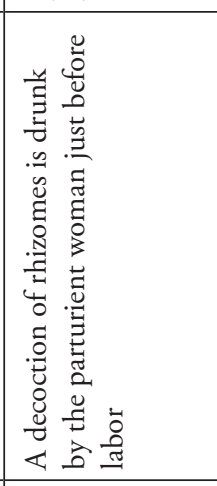 & 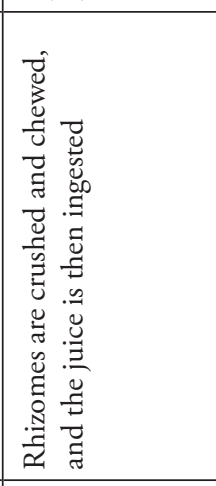 & 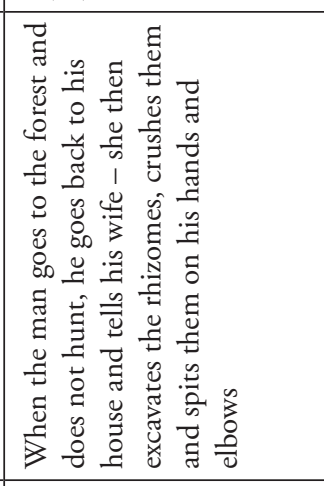 & 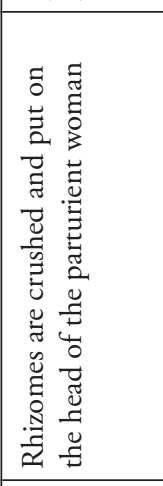 \\
\hline مٌّ & 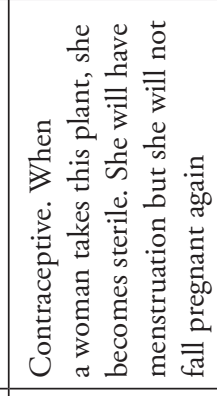 & 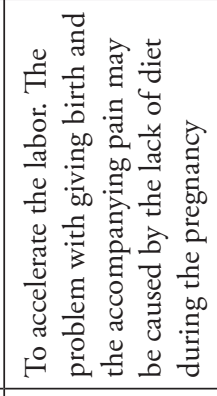 & 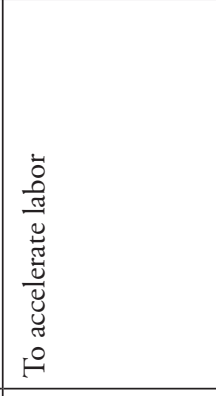 & 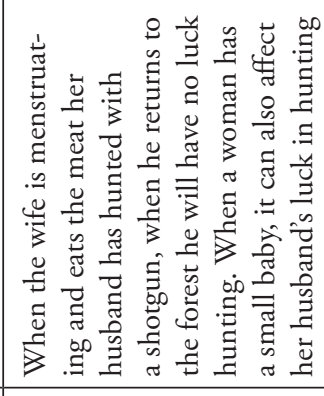 & 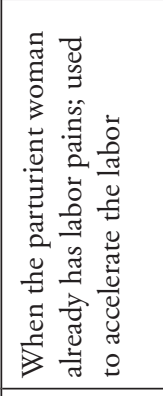 \\
\hline 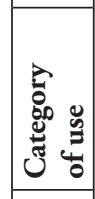 & 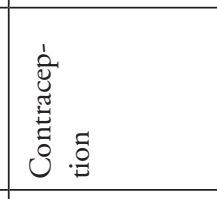 & 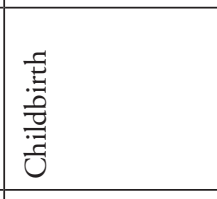 & 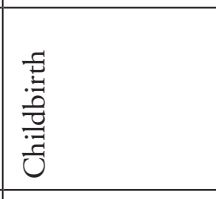 & 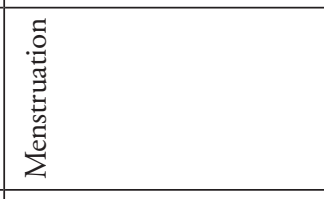 & \\
\hline \begin{tabular}{|l} 
\\
\\
\end{tabular} & 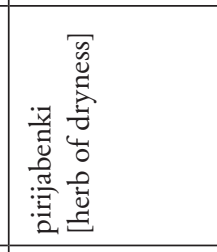 & 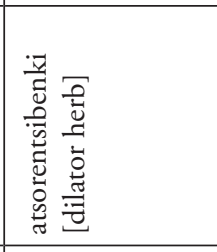 & 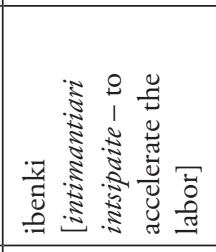 & 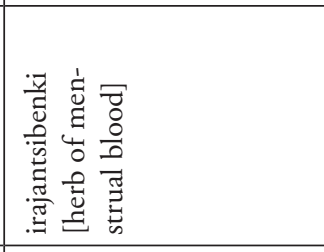 & 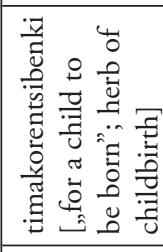 \\
\hline 尊 & 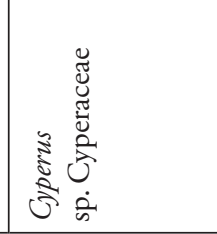 & 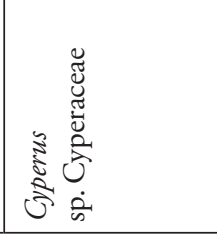 & 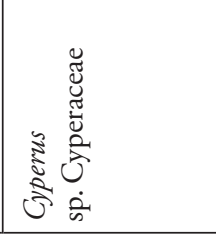 & 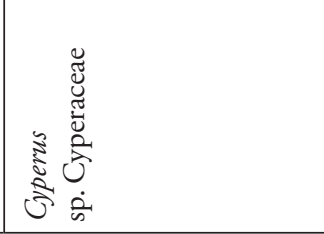 & 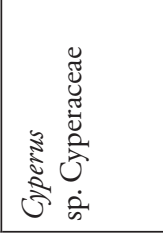 \\
\hline
\end{tabular}




\begin{tabular}{|c|c|c|c|c|c|c|}
\hline 苞 & 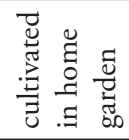 & 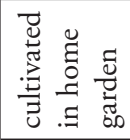 & 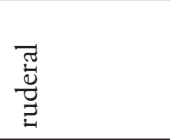 & 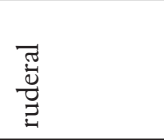 & 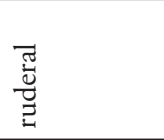 & 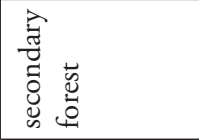 \\
\hline$\stackrel{9}{0}$ & 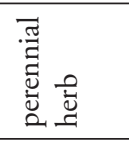 & 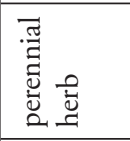 & 氖 & 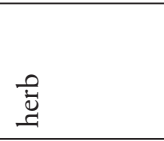 & 竧 & 㤐 \\
\hline 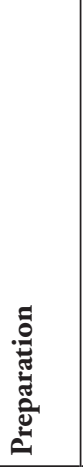 & 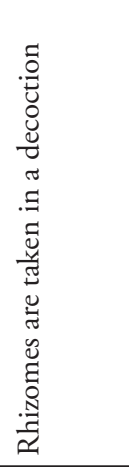 & 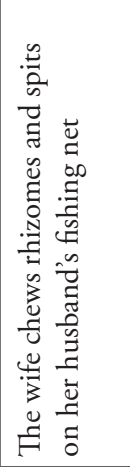 & 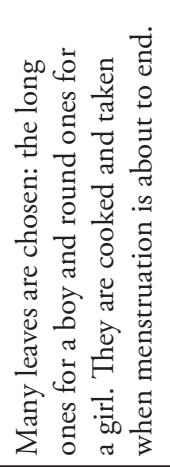 & 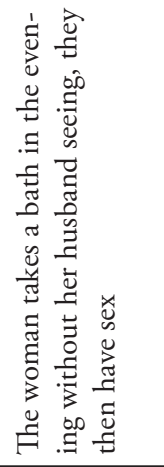 & 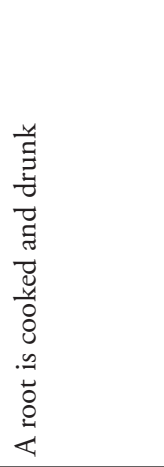 & 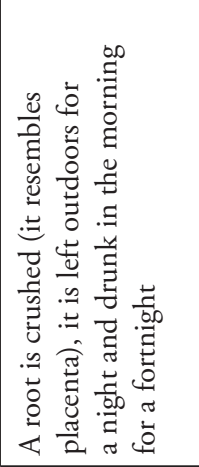 \\
\hline$\stackrel{\infty}{\infty}$ & 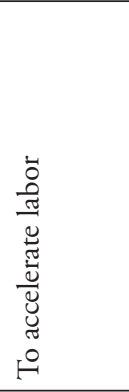 & 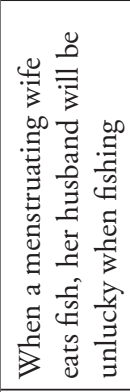 & 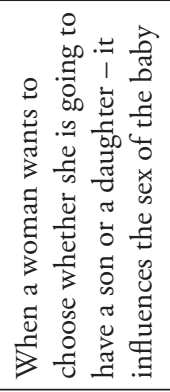 & 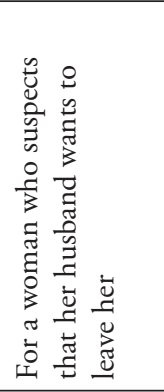 & 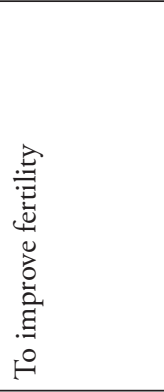 & 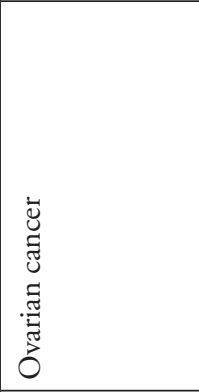 \\
\hline 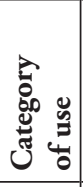 & 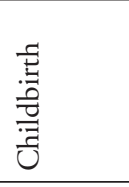 & 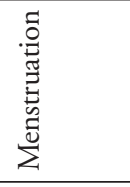 & 胥: & 离 & 点 & 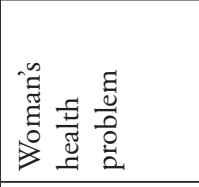 \\
\hline 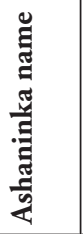 & 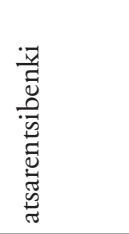 & 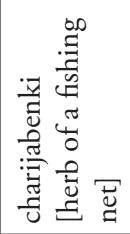 & 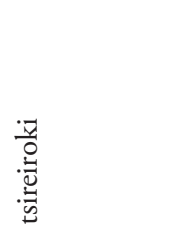 & 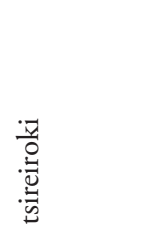 & 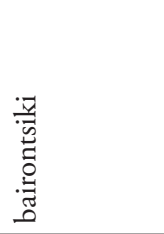 & 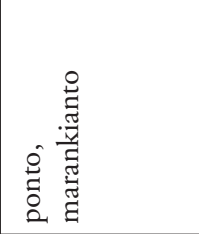 \\
\hline 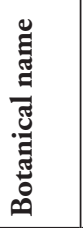 & 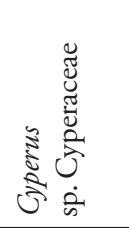 & 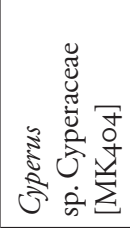 & 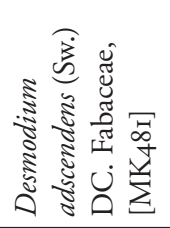 & 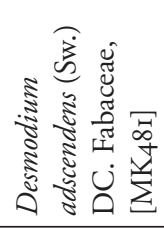 & 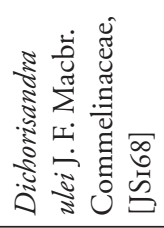 & 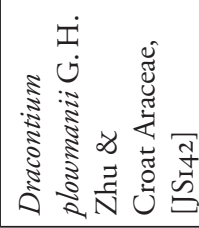 \\
\hline
\end{tabular}




\begin{tabular}{|c|c|c|c|c|c|}
\hline 䔍 & $\begin{array}{l}\overline{\widetilde{g}} \\
\frac{\vec{Z}}{Z}\end{array}$ & 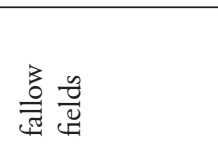 & 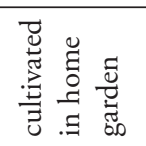 & 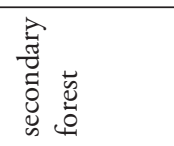 & 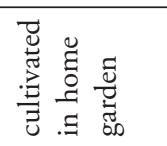 \\
\hline 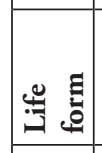 & 咭 & $\stackrel{\text { }}{\stackrel{\Xi}{\Xi}}$ & 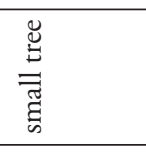 & 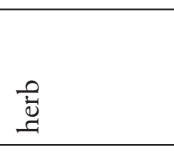 & 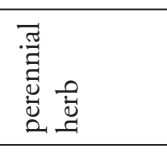 \\
\hline 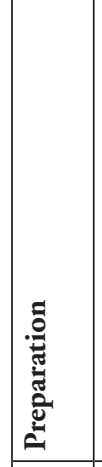 & 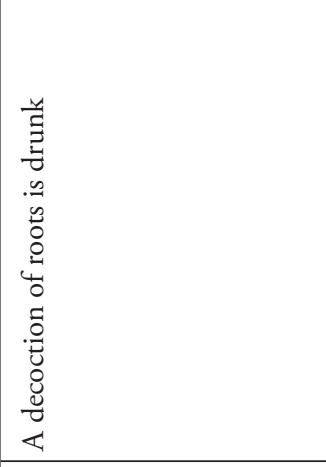 & 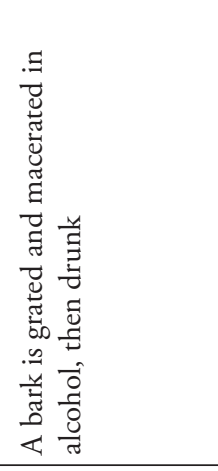 & 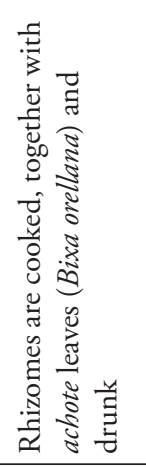 & 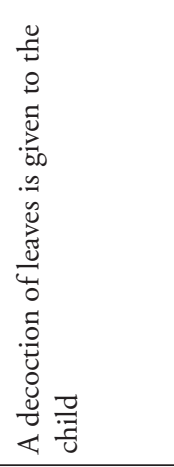 & 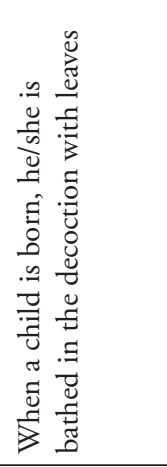 \\
\hline : & 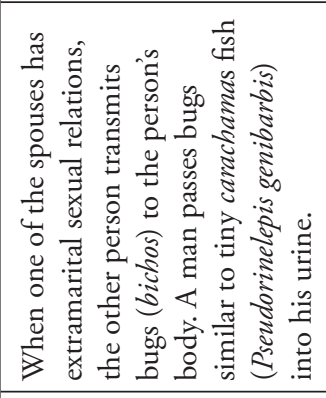 & 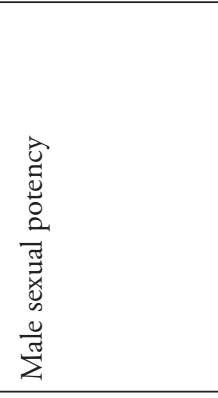 & 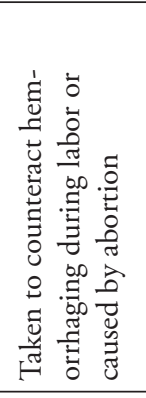 & 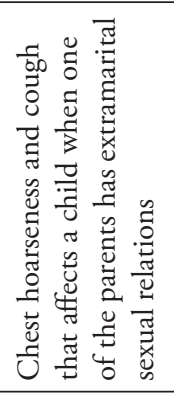 & 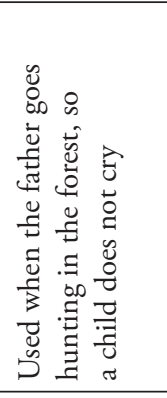 \\
\hline 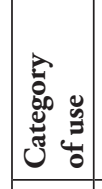 & 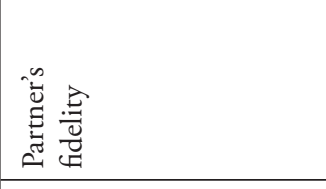 & 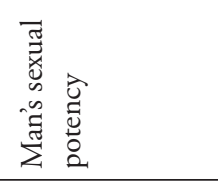 & 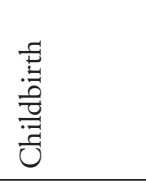 & 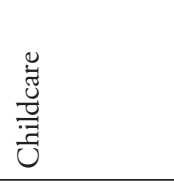 & 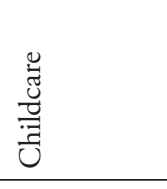 \\
\hline 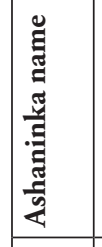 & 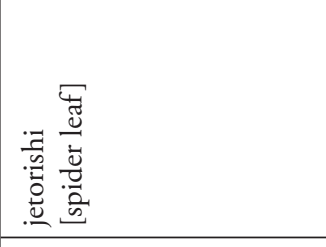 & 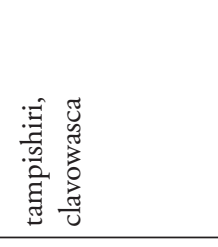 & 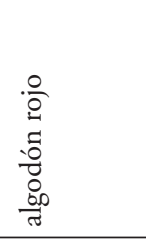 & 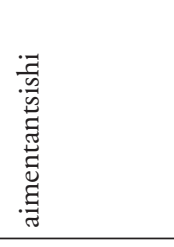 & 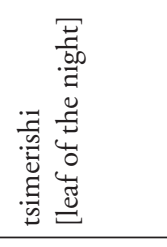 \\
\hline 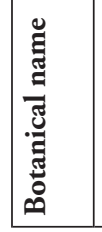 & 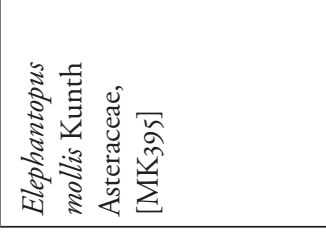 & 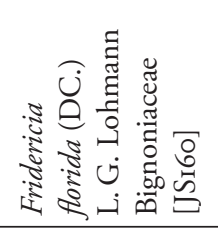 & 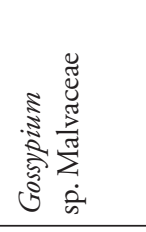 & 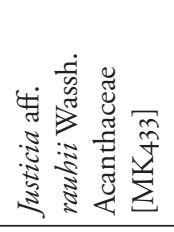 & 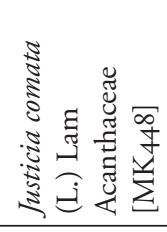 \\
\hline
\end{tabular}




\begin{tabular}{|c|c|c|c|c|c|c|}
\hline 节 & 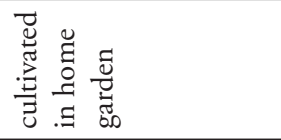 & 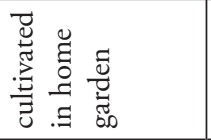 & 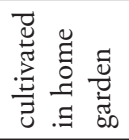 & 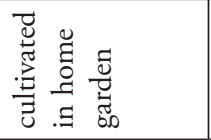 & 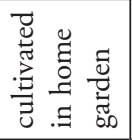 & 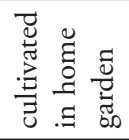 \\
\hline 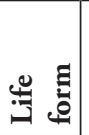 & 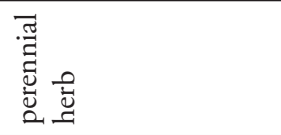 & 咅 & 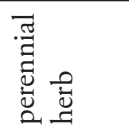 & 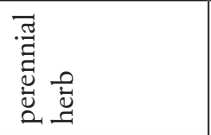 & 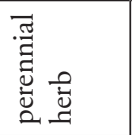 & 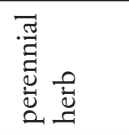 \\
\hline 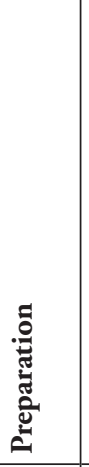 & 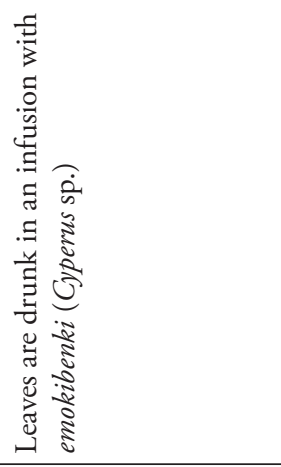 & 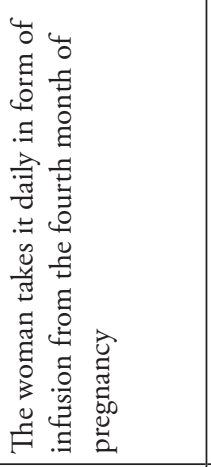 & 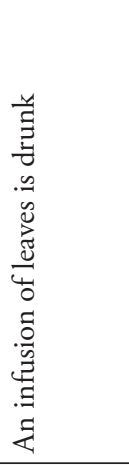 & 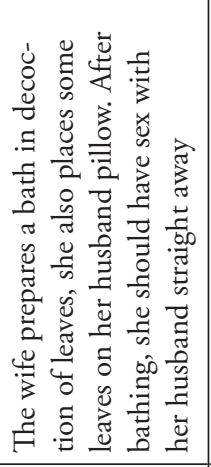 & 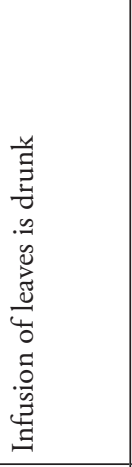 & 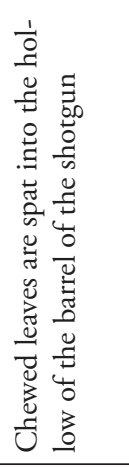 \\
\hline$\ddot{n}$ & 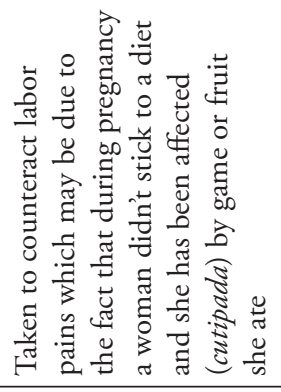 & 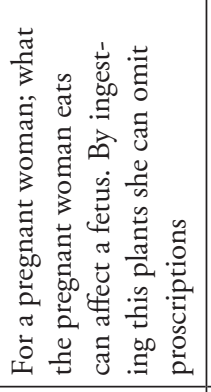 & 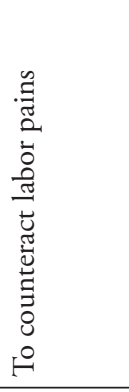 & 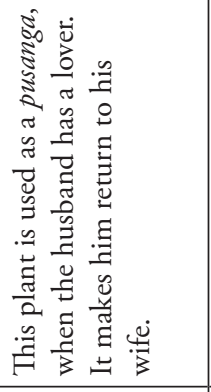 & 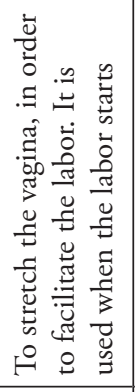 & 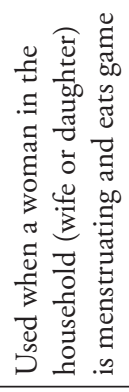 \\
\hline 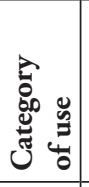 & 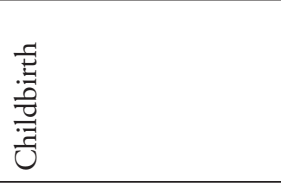 & 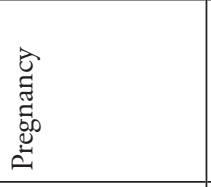 & 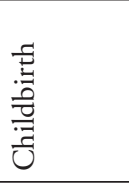 & $\underbrace{\infty}_{\tilde{E}}$ & 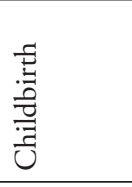 & 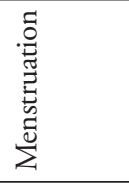 \\
\hline 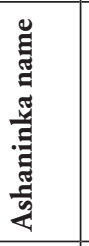 & 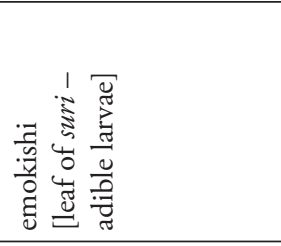 & 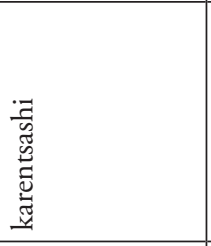 & 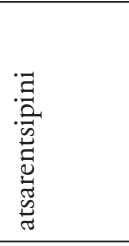 & 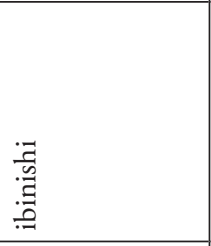 & 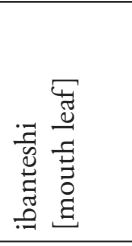 & 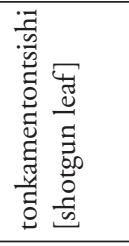 \\
\hline 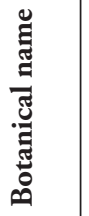 & 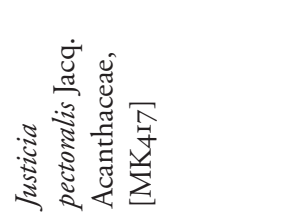 & 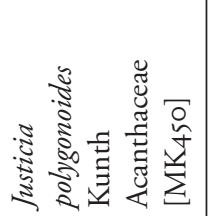 & 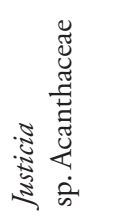 & 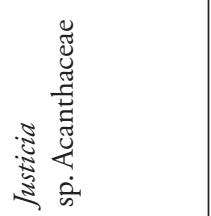 & 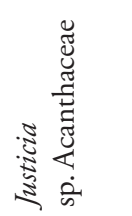 & 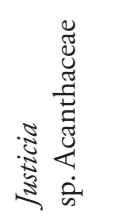 \\
\hline
\end{tabular}




\begin{tabular}{|c|c|c|c|c|c|c|}
\hline 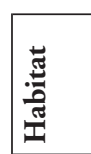 & 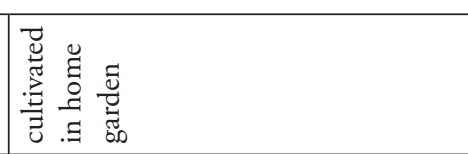 & 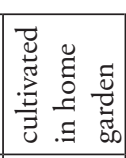 & 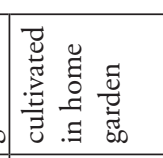 & 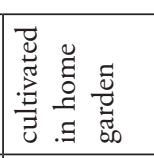 & 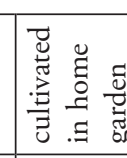 & 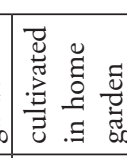 \\
\hline : & 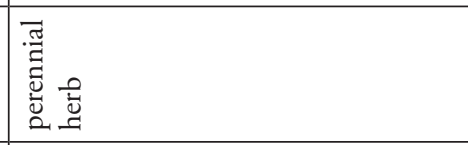 & 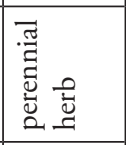 & 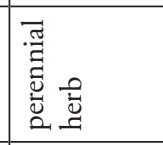 & 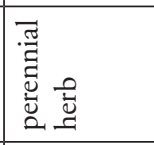 & 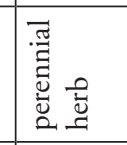 & 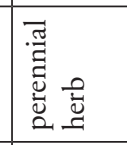 \\
\hline 总 & 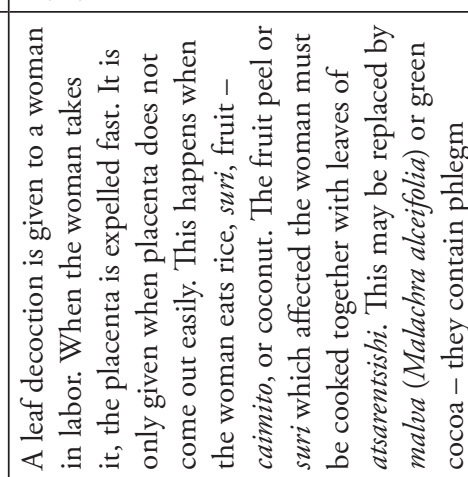 & 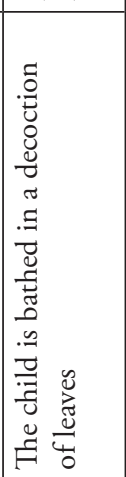 & 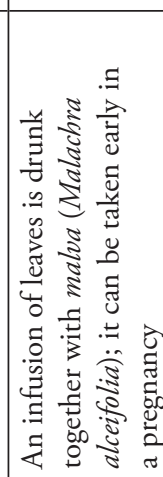 & 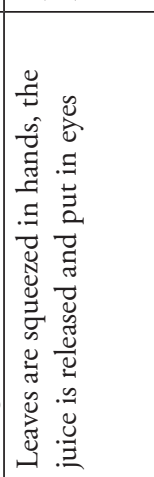 & 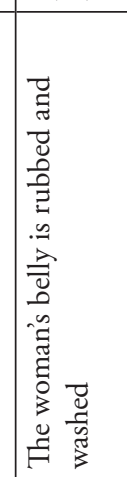 & 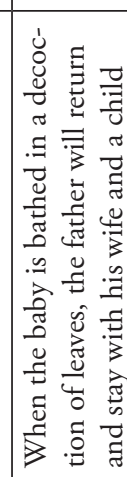 \\
\hline$\stackrel{\mathscr{D}}{2}$ & 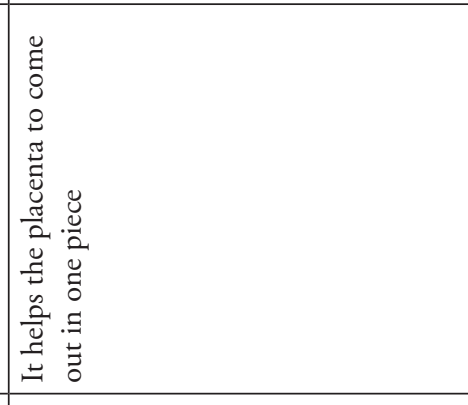 & 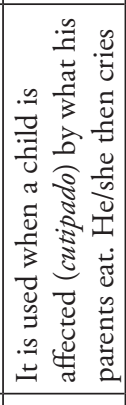 & 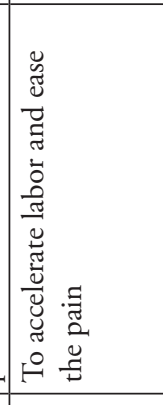 & 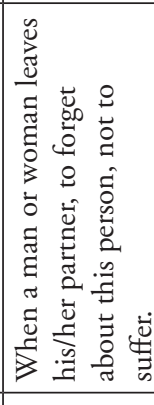 & 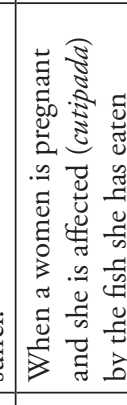 & 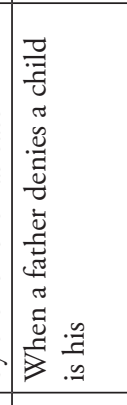 \\
\hline 造 & 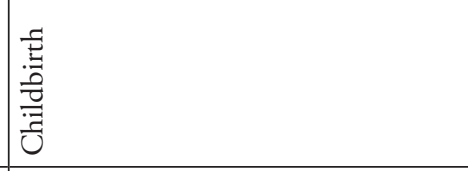 & 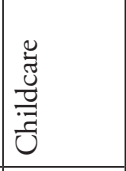 & 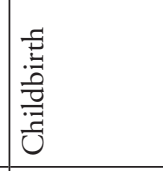 & 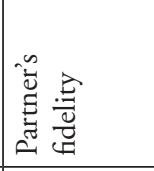 & 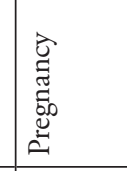 & 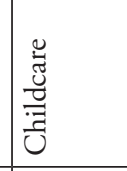 \\
\hline 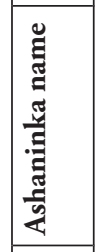 & 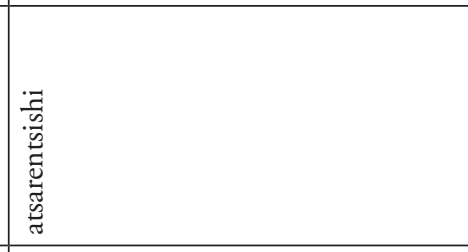 & 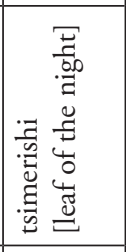 & 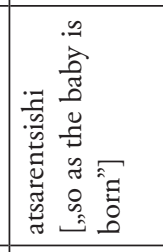 & 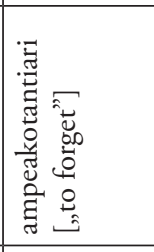 & 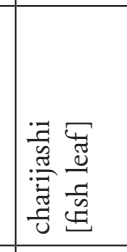 & 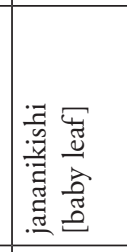 \\
\hline 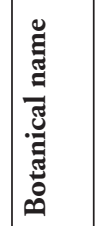 & 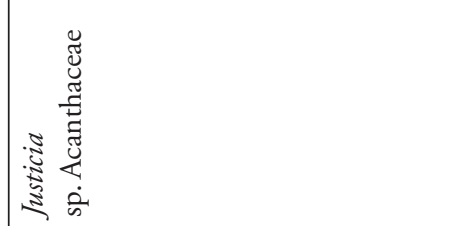 & 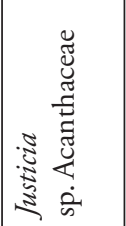 & 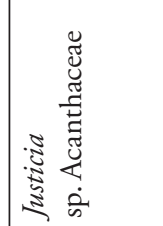 & 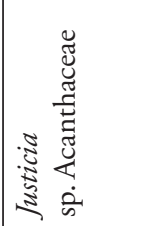 & 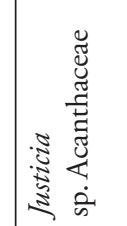 & 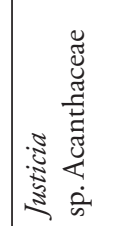 \\
\hline
\end{tabular}




\begin{tabular}{|c|c|c|c|c|c|c|}
\hline 䔍 & 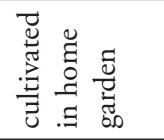 & 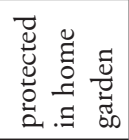 & 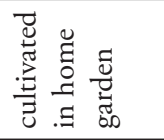 & 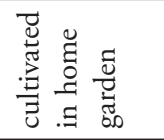 & 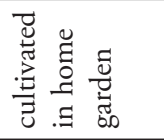 & 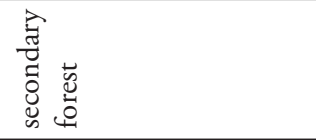 \\
\hline$\stackrel{0}{0}$ & 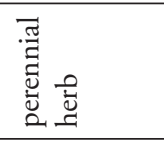 & 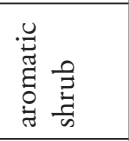 & $\begin{array}{l}\vec{Z} \\
\frac{1}{n}\end{array}$ & $\begin{array}{l}\vec{Z} \\
\frac{\vec{Z}}{\infty} \\
\end{array}$ & $\underset{\Xi}{\Xi}$ & 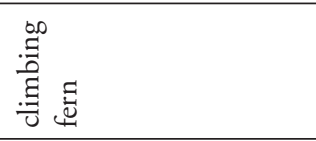 \\
\hline 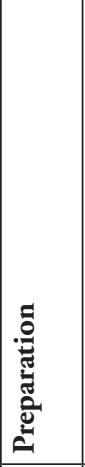 & 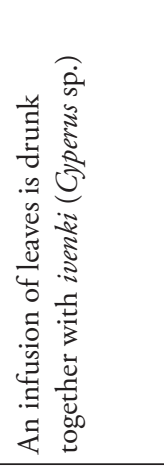 & 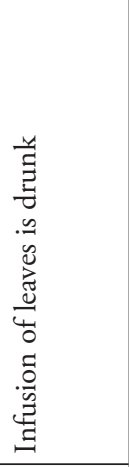 & 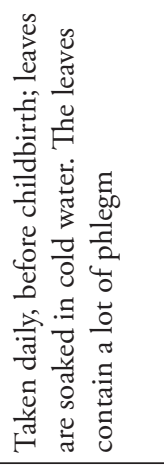 & 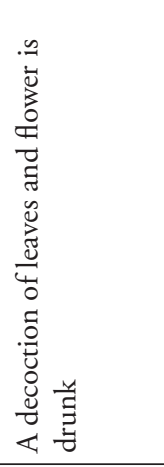 & 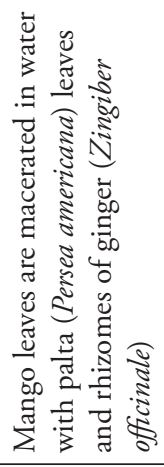 & 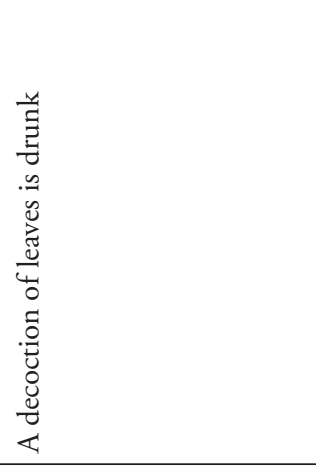 \\
\hline$\stackrel{\mathscr{n}}{.}$ & 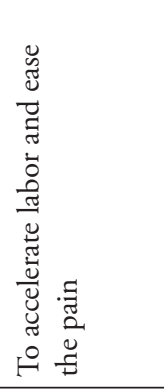 & 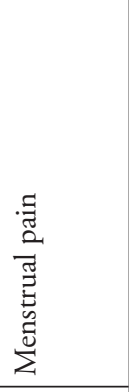 & 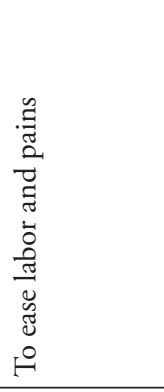 & 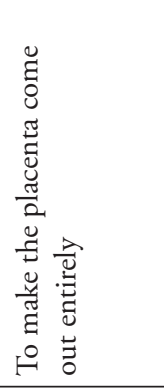 & 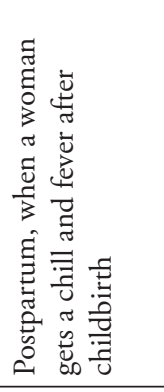 & 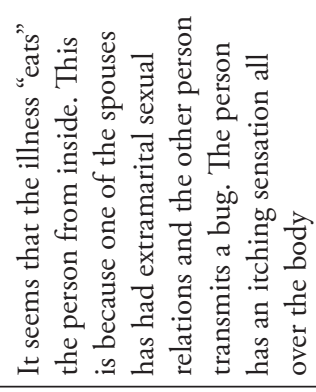 \\
\hline 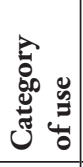 & 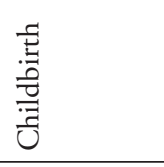 & 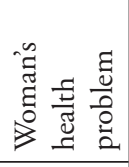 & 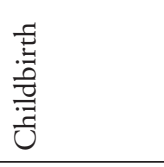 & 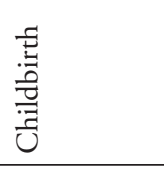 & $\begin{array}{l}\Xi \\
\vdots \\
0 \\
0 \\
2 \\
0 \\
0 \\
0\end{array}$ & 离 \\
\hline 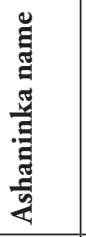 & 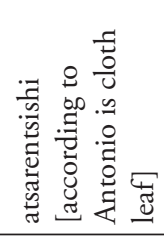 & 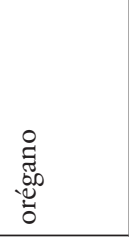 & $\frac{\widetilde{\widetilde{Z}}}{\widetilde{\Xi}}$ & 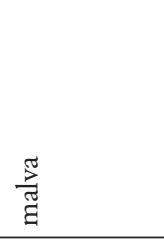 & 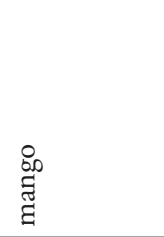 & 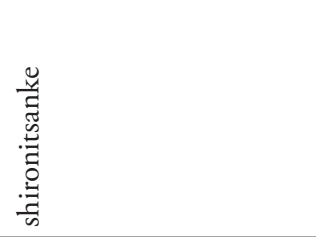 \\
\hline 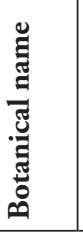 & 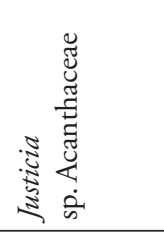 & 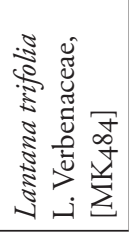 & 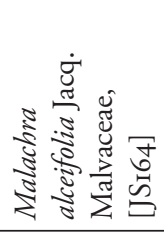 & 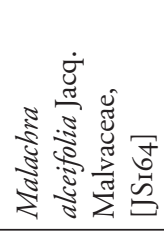 & 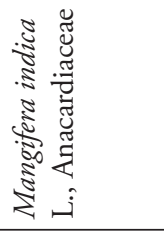 & 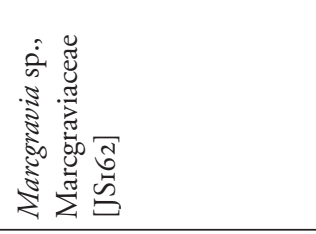 \\
\hline
\end{tabular}




\begin{tabular}{|c|c|c|c|c|c|c|}
\hline 喜 & 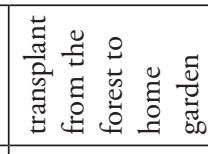 & 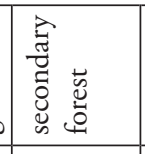 & 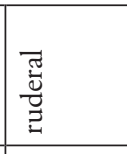 & 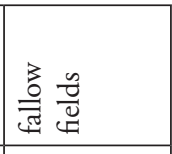 & 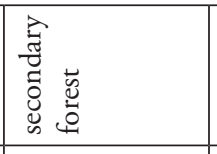 & 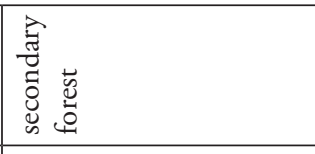 \\
\hline 苛 & 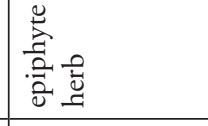 & : & 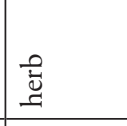 & 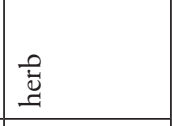 & 离 & 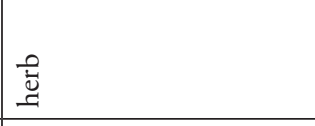 \\
\hline 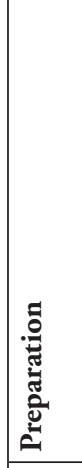 & 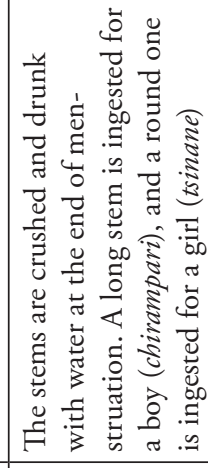 & 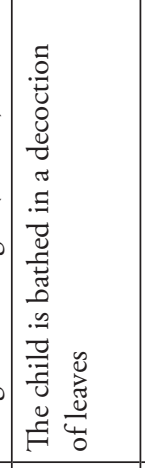 & 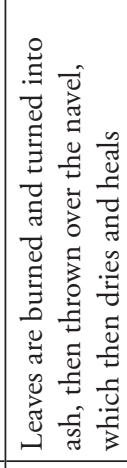 & 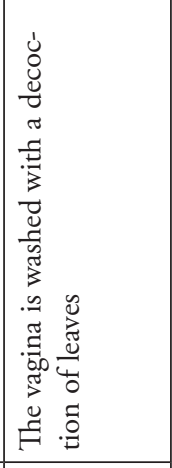 & 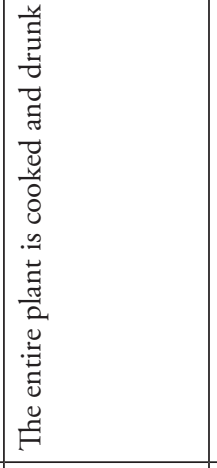 & 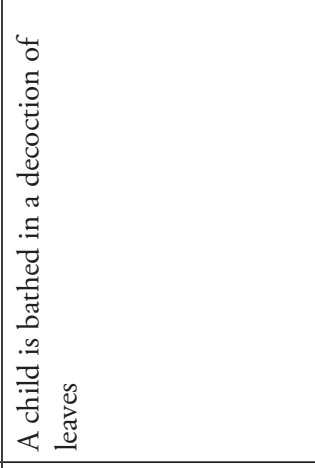 \\
\hline : & 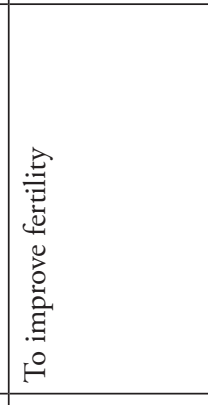 & 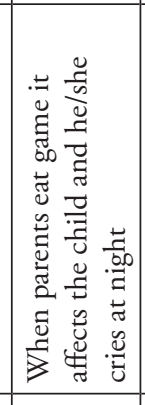 & 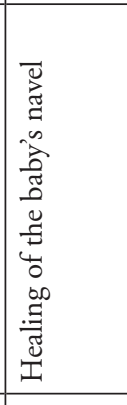 & 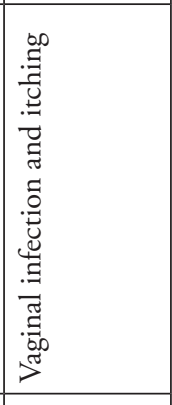 & 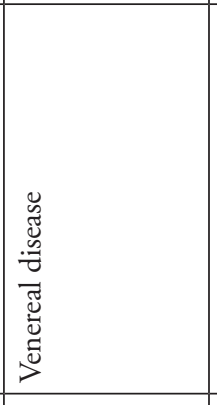 & 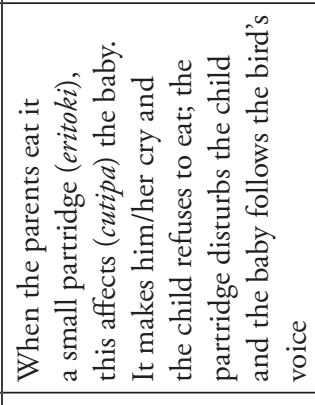 \\
\hline 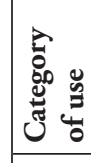 & 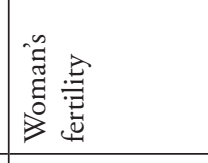 & 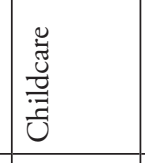 & 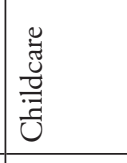 & 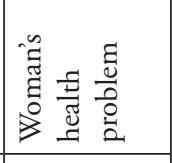 & 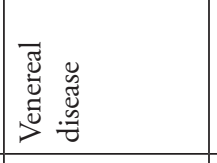 & 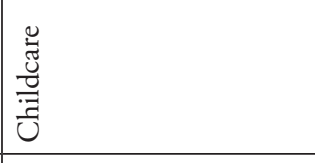 \\
\hline 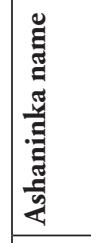 & 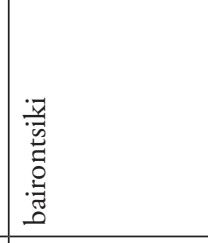 & 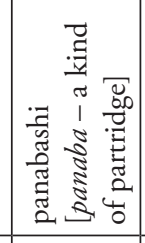 & 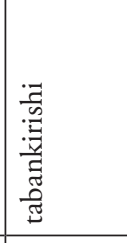 & 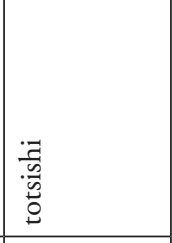 & 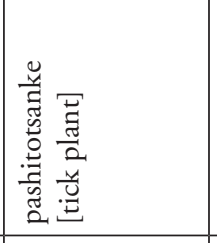 & 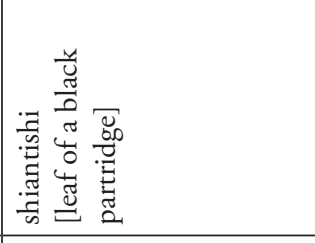 \\
\hline 递 & 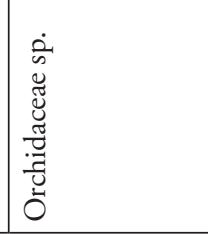 & 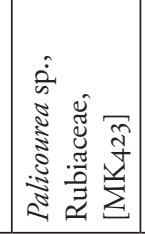 & 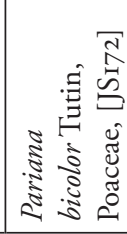 & 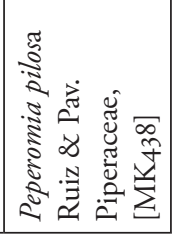 & 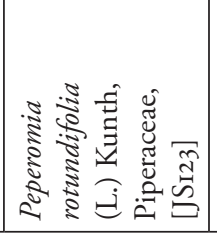 & 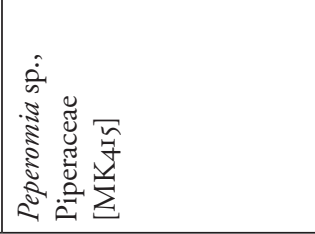 \\
\hline
\end{tabular}




\begin{tabular}{|c|c|c|c|c|c|}
\hline 节 & 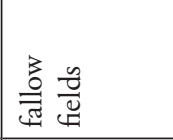 & 亭 $\frac{n}{\mathbb{E}}$ & 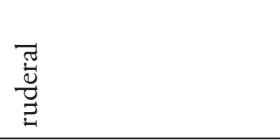 & 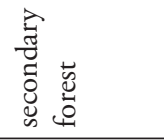 & 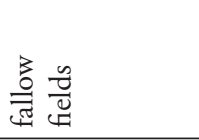 \\
\hline 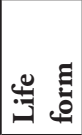 & 竘 & $\begin{array}{l}0 \\
\vec{E} \\
\text { ज } \\
0 \\
0\end{array}$ & 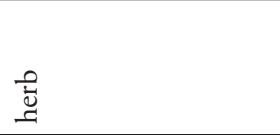 & 竞 & $\frac{0}{5}$ \\
\hline 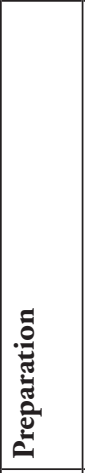 & 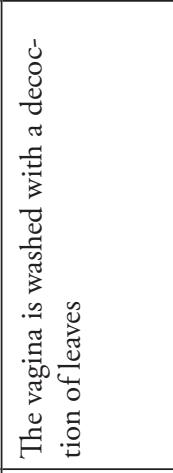 & 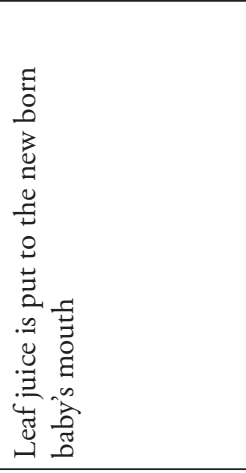 & 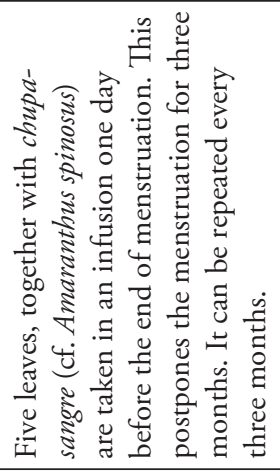 & 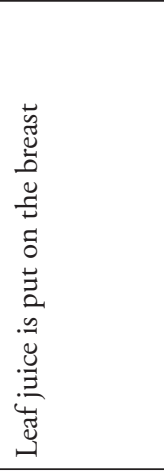 & 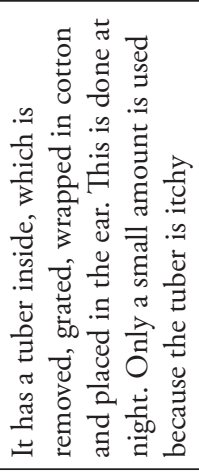 \\
\hline$\stackrel{\mathscr{n}}{\omega}$ & 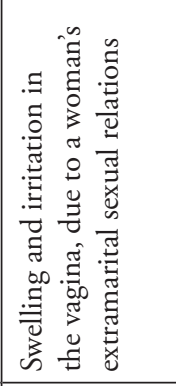 & 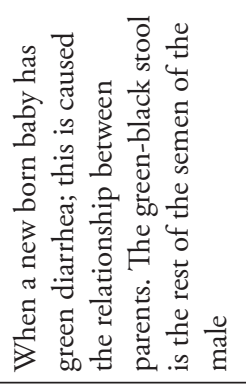 & 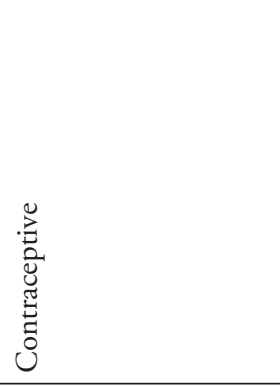 & 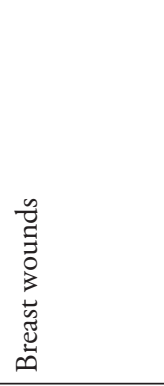 & 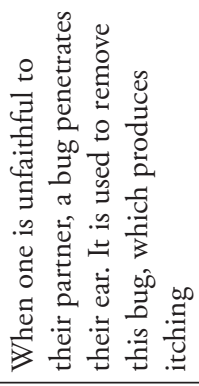 \\
\hline 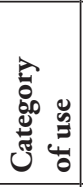 & 离 & 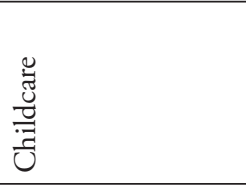 & 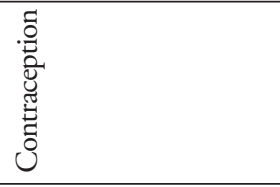 & 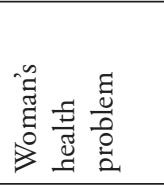 & 离总 \\
\hline 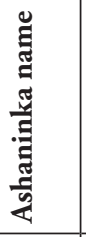 & 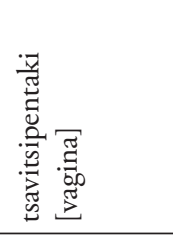 & 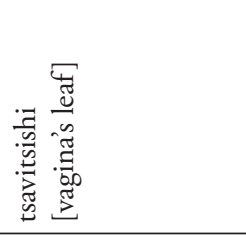 & 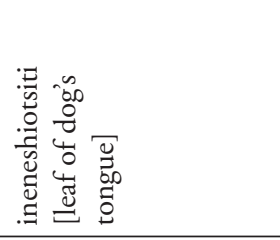 & 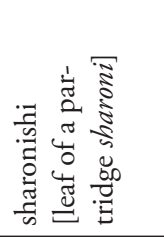 & 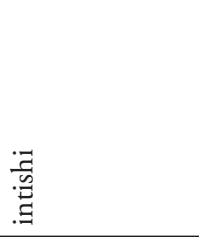 \\
\hline 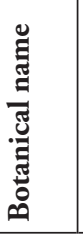 & 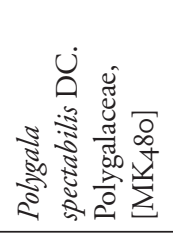 & 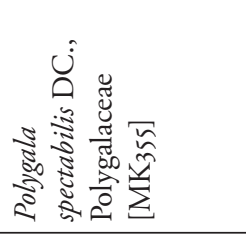 & 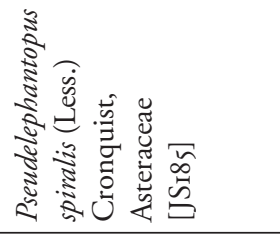 & 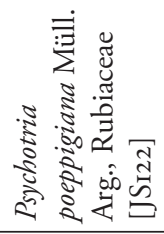 & 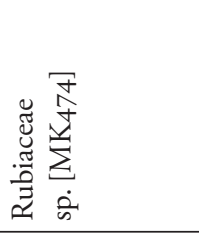 \\
\hline
\end{tabular}




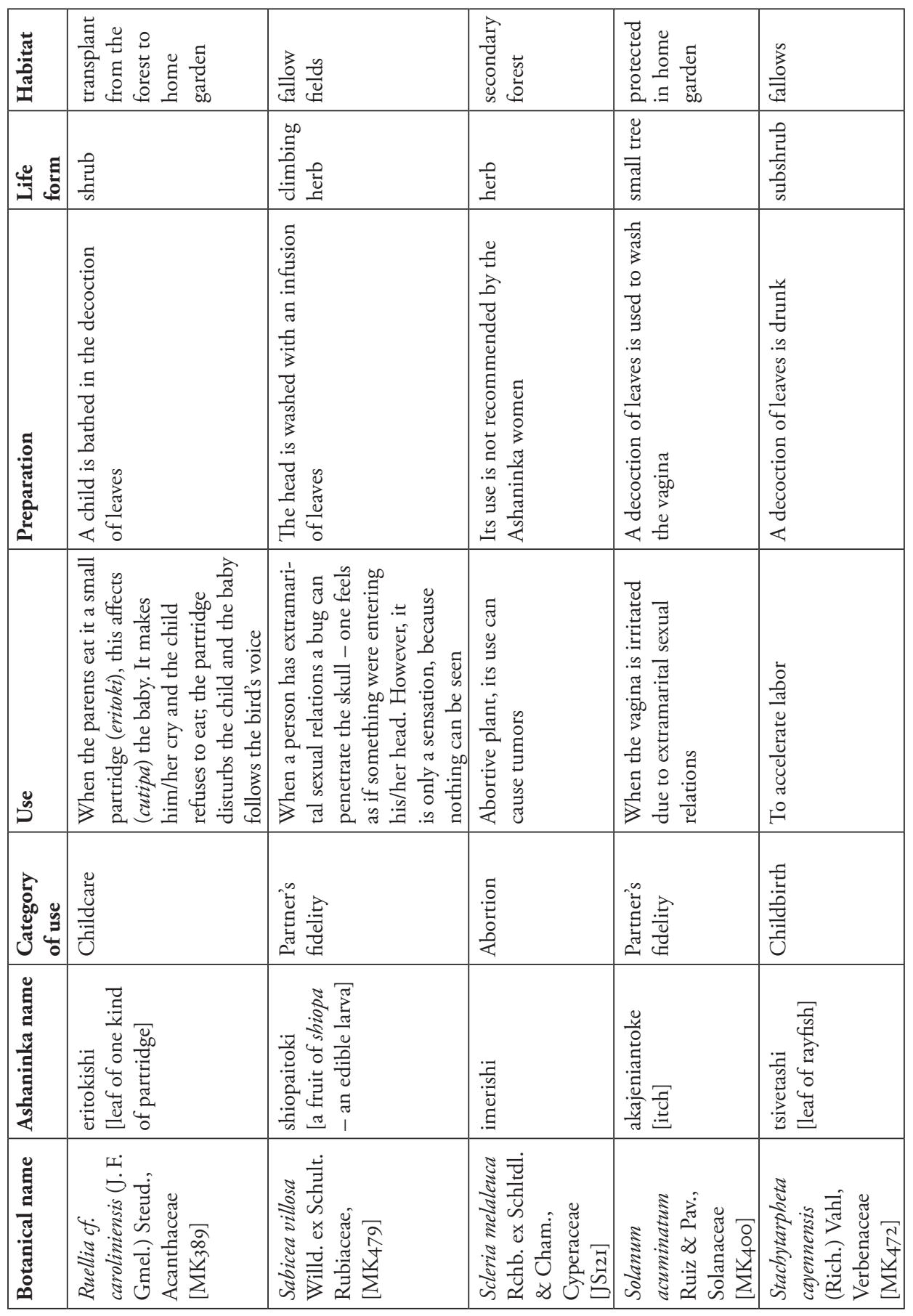




\begin{tabular}{|c|c|c|c|c|}
\hline 葋 & 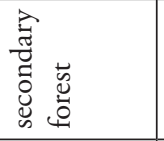 & 亮 & $\begin{array}{l}\overline{\widetilde{d}} \\
\frac{\vec{v}}{\vec{Z}} \\
\end{array}$ & 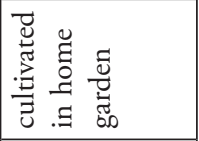 \\
\hline$\stackrel{5}{0}$ & E & $\underset{\Xi}{\Xi}$ & $\begin{array}{l}\text { 咅 } \\
\text { 至 }\end{array}$ & 氞 \\
\hline 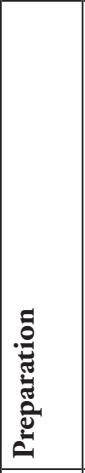 & 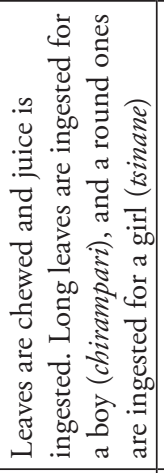 & 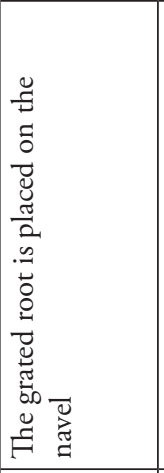 & 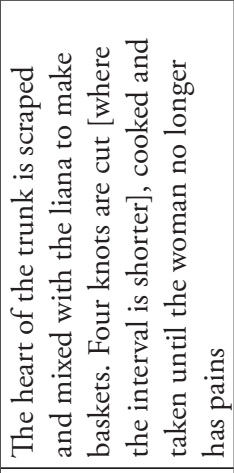 & 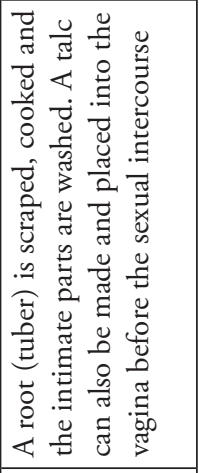 \\
\hline$\stackrel{\mathscr{D}}{D}$ & 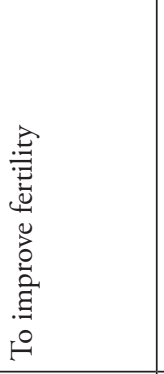 & 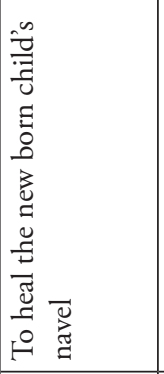 & 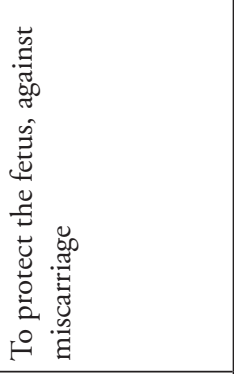 & 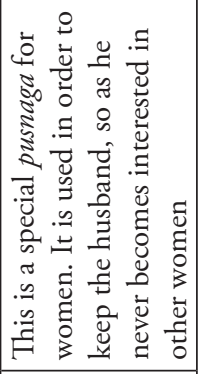 \\
\hline 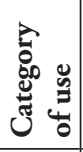 & 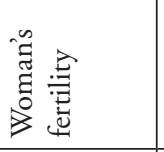 & 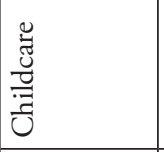 & 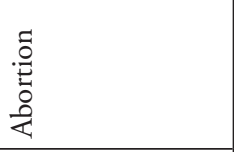 & 离离 \\
\hline 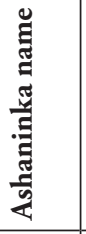 & 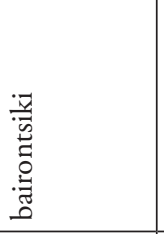 & 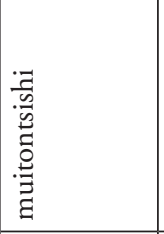 & $\stackrel{\circ}{\stackrel{0}{a}}$ & 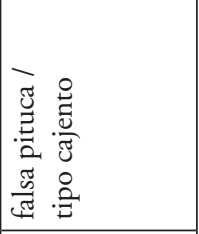 \\
\hline 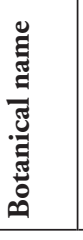 & 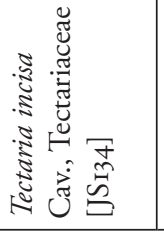 & 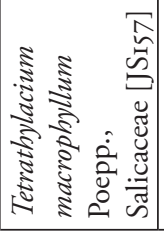 & 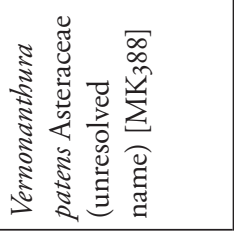 & 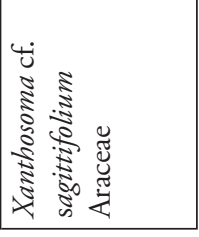 \\
\hline
\end{tabular}




\section{BIBLIOGRAPHY}

$\AA ̊$ r h e m K. 1996. The Cosmic Food Web. Human-nature Relatedness in the Northwest Amazon. In P. Descola and G. Pálsson (eds.), Nature and Society: Anthropological Perspective. London, 185-204.

B a e r H. B. 2003. Contribution to a Critical Analysis of Medical Pluralism: an Examination of the Work of Libbet Crandon-Malamud. In J. Koss-Chioino, T. Leathermand and C. Greenway (eds.), Medical Pluralism in the Andes. London, 42-60.

B ela und e L.E. 1997. 'Looking after your woman': Contraception amongst the Airopai (Secoya) of Western Amazonia. Anthropology \& Medicine 4, I3I-I44.

Carus o E. 20II. Co-management Redux: Anti-politics and Transformation in the Ashaninka Communal Reserve, Peru. International Journal of Heritage Studies 17, 608-628.

Ca y ón L. 2013. Pienso luego creo. La teoría makuna del mundo. Bogotá.

$\mathrm{C}$ rand o n-M l a m u d L. I991. From the Fat of our Souls: Social Change, Political Process, and Medical Pluralism in Bolivia. Berkeley.

De Burgos H. 20I4. Contemporary Transformations of Medicine and Ethnic Identity. Anthropologica 56 (2), 399-413.

Descola P. 1996. Constructing Natures. Symbolic Ecology and Social Practice. In P. Descola and G. Pálsson (eds.), Nature and Society: Anthropological Perspective. London and New York, 83-102.

D e s cola P. 2013. Beyond Nature and Culture. Chicago.

Freed W.J., Gilli n J. C., Wyat t R. J. 1979. Anticonvulsant Properties of Betaine. Epilepsia 20, 209-2I3.

Girardi C., Fabre N., Paloque L., Pramundita Ramadani A., Benoit-Vical F., González-Aspejo G., Haddad M., Rengifo E., Jullian V. 20I5. Evaluation of Antiplasmodial and Antileishmanial Activities of Herbal Medicine Pseudelephantopus spiralis (Less.) Cronquist and Isolated Hirsutinolide-type Sesquiterpenoids. Journal of Ethnopharmacology $170, \mathrm{I} 67-\mathrm{I} 74$.

Gold C.L. and Cla p p A. 2orr. Negotiating Health and Identity: Lay Healing, Medicinal Plants, and Indigenous Healthscapes in Highland Peru. Latin American Research Review 46 (3), 93-III.

Heckenberger M. 2005. The Ecology of Power: Culture, Place, and Personhood in the Southern Amazon, AD I000-2000. New York.

He ckle r S. L. 2007. Herbalism, Home Gardens and Hybridization. Wôthïhã Medicine and Cultural Change. Medical Anthropology Quarterly 2I, 4I-63.

Her n W.M. 1994. Alta fecundidad en una comunidad nativa de la Amazonia Peruana. Amazonía Peruana I2, I25-I42.

Holling C.S. 20or. Understanding the Complexity of Economic, Ecological, and Social Systems. Ecosystems 4, 390-405.

I z qu i e rd o C. When "Health" Is Not Enough: Societal, Individual and Biomedical Assessments of Well-being among the Matsigenka of the Peruvian Amazon. Social Science \& Medicine 6I, 767-783.

Joh annessen H. and Lázá r I. (eds.) 2006. Multiple Medical Realities. Patients and Healers in Biomedical, Alternative and Traditional Medicine. New York.

J o h n s o n A. 2003. Families of the Forest: the Matsigenka Indians of the Peruvian Amazon. Berkeley.

Keck M. and Sakdapolrak P. 20I3. What is Social Resilience? Lessons Learned and Ways Forward. Erdkunde 67, 5-19.

Killick E . 2009. Creating Community. Land Titling, Education and Settlement Formation among the Ashéninka of Peruvian Amazonia. Journal of Latin American and Caribbean Anthropology 13, 22-47.

Le ipert B. D. and Reuter L. 2005. Developing Resilience: How Women Maintain their Health in Northern Geographically Isolated Settings. Qualitative Health Research I5, 49-65. 
Len a erts M. 2006. Substances, Relationships and the Omnipresence of the Body: An Overview of Ashéninka Ethnomedicine (Western Amazonia). Journal of Ethnobiology and Ethnomedicine 2: 49.

Macra e W.D. and Towe rs G. H. N. 1984. Justicia pectoralis: The Study of the Basis for its Use as a Hallucinogenic Snuff Ingredient. Journal of Ethnopharmacology I2, 93-III.

Maxwell N. 1972. Actitudes de cuatro tribus de la selva peruana respecto a plantas empleadas como anticonceptivos por vía oral. Historia, etnohistoria y etnología de la selva sudamericana. Actas y Memorias del XXXIX Congreso Internacional de Americanistas. Lima, IOO-IO7.

Monten egro R.A. and Stephens C. 2006. Indigenous Health in Latin America and the Caribbean. The Lancet 367, 1859-1869.

O brist B., May u m a na I., Kes s y F. 20Io. Livelihood, Malaria and Resilience: a Case Study in Kilombero Valley, Tanzania. Progress in Development Studies I0, 325-343.

Plow man T.C., Le u chtmann A., B l a n e y C., Clay K. 1990. Significance of the Fungus Balansia cyperi Infecting Medicinal Species of Cyperus (Cyperaceae) from Amazonia. Economic Botany 44 (4), 452-462.

Rival L. M. 1998. Androgynous Parents and Guest Children: the Huaorani Couvade. The Journal of the Royal Anthropological Institute 4 (4), 619-642.

Riv a l L. M. 2002. Trekking Through History. The Huaorani of Amazonian Ecuador. New York.

S a hl in s M. 1995. Historical Metaphor and Mythical Realities: Structure in the Early History of Sandwich Islands. ASAO Special Publication, Ann Arbor.

S e s i a P. M. 1996. 'Women Come Here on Their own when They Need to': Parental Care, Authoritative Knowledge, and Maternal Health in Oaxaca. Medical Anthropology Quarterly IO, I2I-I40.

She pard Jr. G. H. 1998. Psychoactive Plants and Ethnopsychiatric Medicines of the Matsigenka. Journal of Psychoactive Drugs 30, 321-332.

Varese S. 2006. La sal de los cerros. Lima.

Viveiros de Castro E. 1998. Cosmological Deixes and Amerindian Perspectivism. The Journal of the Royal Anthropological Institute 4 (3), 469-488.

Zank S., Araujo de L.G., Hanazaki N. 20I9. Resilience and Adaptability of Traditional Healthcare System: A Case Study of Communities in Two Regions of Brazil. Ecology and Society (online first/without pagination).

\section{List of table and figure captions}

Figure I. Leaves of Xanthosoma cf. sagittifolium which resemble vagina. The starchy tuber of this plant is used as a pusanga (photo: M. Kujawska).

Figure 2. Leaves of ohoairontsiki (Tectaria incise) used to improve women's fertility. They are consumed in a way that a woman may choose the sex of the baby: when long leaves are ingested, it is for a boy (chirampari) and whereas round leaves, it will result in a girl being born (tsinane) (photo: M. Kujawska).

Table I. Medicinal plants used in reproductive health by Ashaninka people from the Tambo river valley.

\section{Author's address}

Monika Kujawska PhD

Department of Ethnology and Cultural Anthropology

University of Lodz

ul. Lindleya 3/5, 90-I3 Łódź, POLAND

e-mail: monika.kujawska@uni.lodz.pl

ORCID: 0000-0002-5643-34I7 